\title{
Jucys-Murphy elements for Soergel bimodules
}

\author{
STEEN RYOM-HANSEN*
}

\begin{abstract}
We produce Jucys-Murphy elements for the diagrammatical category of Soergel bimodules associated with general Coxeter groups, and use them to diagonalize the bilinear form on the cell modules. This gives rise to an expression for the determinant of the forms and Jantzen type sum formulas.
\end{abstract}

\section{Introduction}

Soergel bimodules were introduced by Soergel around 1990 in order to prove the Koszul duality conjectures for category $\mathcal{O}$ of a complex simple Lie algebra. At the same time they also gave rise to a new proof of the Kazhdan-Lusztig conjectures for the composition factor multiplicities of Verma modules. Although Soergel bimodules are essentially combinatorial objects defined in terms of the corresponding Weyl group, the proof of these conjectures relied on intersection homology methods.

Some time later Soergel realized that the bimodule theory makes sense for arbitrary Coxeter systems $(W, S)$ and formulated in this general context the Soergel conjecture, relating the indecomposable bimodules with the Kazhdan-Lusztig basis of the associated Hecke algebra. This conjecture implies the positivity conjectures for Kazhdan-Lusztig polynomials. It was recently proved in a celebrated work by Elias and Williamson. Their proof was entirely algebraic and even gave rise to a proof of the Kazhdan-Lusztig conjectures about composition factors of Verma modules that avoids intersection homology.

In positive characteristic and for $W$ infinite, the category of Soergel bimodules does not categorify the Hecke algebra associated with $(W, S)$ and the relationship to representation theory becomes unclearer. But over the last decade, starting with the work of Elias and Khovanov, a diagrammatical category $\mathcal{D}$ has been developed that remedies this problem. Apart from the theoretical advantages of $\mathcal{D}$ over the bimodule category, certain calculations are more accessible in $\mathcal{D}$ than in the bimodule category. Indeed Williamson's counterexamples to the explicit bound given in Lusztig's famous conjecture for the representation theory of algebraic groups in characteristic $p$ used calculations involving $\mathcal{D}$.

In this paper we study the diagrammatical category $\mathcal{D}$ starting from the fact, proved by Elias and Williamson, that $\mathcal{D}$ is a cellular category. The cellular basis is here a diagrammatical adaption of Libedinsky's light leaves.

The cellular algebra approach to the representation theory of the symmetric group and Hecke algebra in type $A$ was pioneered by Murphy. One of his major insights was the proof that the classical Jucys-Murphy elements $\left\{L_{i}\right\}$ act lower triangularly on the cellular basis in these cases. To a large degree it is this triangularity property that explains the importance of the Jucys-Murphy elements in modern representation theory. In fact, it has been axiomatized by Mathas as the defining property of a cellular algebra endowed with a family of JM-elements.

The objects of $\mathcal{D}$ are expressions over $S$, that is words in the alphabet $S$, and for each such expression $\underline{w}$ we consider the endomorphism algebra $A_{\underline{w}}=\operatorname{End}_{\mathcal{D}}(\underline{w})$. It is a cellular algebra in the original sense of Graham and Lehrer. The first main result of the paper is our Theorem 5.2 stating that $A_{\underline{w}}$ is a cellular algebra endowed with a family of JM-elements, in the sense of Mathas. Apart

* Supported in part by FONDECYT grant 1171379 
from the intrinsic value of this result, the JM-elements are of interest by themselves. They are of the form

$$
L_{i}=|| \cdots+\ldots \mid
$$

and already appear in the literature. For example, whenever $\underline{w}$ corresponds to a reduced expression of an element of $W$, the Lefschez operator $\rho$ from Elias and Williamson's proof of Soergel's conjecture can be written as a linear combination with coefficients in $\mathbb{R}^{+}$of these $L_{i}$ 's. We hope that this connection between $\rho$ and the $L_{i}$ 's can be the inspiration for the construction of Lefschez operators for other $\mathbb{Z}$-graded representation theories that arise in algebraic Lie theory.

We are interested in the representation theory of the cellular algebras $A_{\underline{w}}$. By the general theory for cellular algebras, for each $A_{\underline{w}}$ there exists a family of cell modules $\Delta_{\underline{w}}(\bar{y})$, each endowed with a bilinear form $\langle\cdot, \cdot\rangle_{y}$ such that the simple $A_{\underline{w}}$-modules are of the form $L_{\underline{w}}(y)=\Delta_{\underline{w}}(y) / \operatorname{rad}\langle\cdot, \cdot\rangle_{y}$. In our particular case $y$ runs over those elements of $W$ that appear as a subexpression of $\underline{w}$.

In fact, $\mathcal{D}$ is a $\mathbb{Z}$-graded cellular category and so $A_{\underline{w}}$ becomes a $\mathbb{Z}$-graded cellular algebra in the sense of $\mathrm{Hu}$ and Mathas. In the case of the ground field $\mathbb{R}$, the representation theory of $A_{\underline{w}}$ was studied by D. Plaza who showed that its associated graded decomposition numbers are the KazhdanLusztig polynomials associated with $(W, S)$. In characteristic $p$ the decomposition numbers are the $p$-Kazhdan-Lusztig polynomials.

One of the important applications of the triangularity property of the Jucys-Murphy elements, first due to James and Murphy for the symmetric group, is to diagonalize the bilinear form. This gave rise to a cancellation free expression for the determinant of the form. In this paper we show that James and Murphy's idea can be carried out in the setting of $\mathcal{D}$ as well, even though the combinatorics of $\mathcal{D}$ is very different from the Young diagram combinatorics of the symmetric group, of course. Our formula, given in Theorem 8.5 of the paper, is the following one

$$
\operatorname{det}\langle\cdot, \cdot\rangle_{y}= \pm \prod_{\beta>0, s_{\beta} y>y} \beta^{\operatorname{dim} \Delta\left(s_{\beta} y\right)}
$$

where $\beta>0$ means that $\beta$ is positive root for $W$ : we must here impose conditions for this notion to make sense. Note that the formula has much resemblance with the classical Shapovalov formula for Verma modules.

Following Jantzen's original ideas for Verma modules, we next go on to construct a filtration of $A_{\underline{w}}$-submodules $\Delta_{\underline{w}}(y)=\Delta_{\underline{w}}^{0}(y) \supseteq \Delta_{\underline{w}}^{1}(y) \supseteq \Delta_{\underline{w}}^{2}(y) \supseteq \ldots$ on $\Delta_{\underline{w}}(y)$ and wish to deduce from (1.2) a sum formula for this filtration valid in the Grothendieck group. Here the lack of quasi-heredity of $A_{\underline{w}}$ turns out to be an obstacle for a direct translation of Jantzen's ideas to our setting, since the $\Delta_{\underline{w}}(y){ }^{\prime}$ s do not induce a basis of the corresponding Grothendieck group. We resolve this problem by replacing $\underline{w}$ by a certain subset $\underline{\pi}$ of the expressions over $S$, containing $\underline{w}$, and satisfying that the corresponding subset $\pi \subseteq W$ is an ideal in $W$ with respect to the Bruhat order. This gives rise to another cellular algebra $A_{\underline{\pi}}$ with cell modules $\Delta_{\underline{\pi}}(y)$. The algebra $A_{\underline{\pi}}$ is quasi-hereditary as we show in Theorem 8.5 , and therefore solves the above mentioned problem.

The above results are valid for any ground field $\mathbb{k}$ such that char $\mathbb{k}>3$ and so for any valuation $\nu$ on the ground ring $R$ whose fraction field is $\mathbb{k}$ we obtain a filtration $\Delta_{\underline{\underline{\pi}}}(y)=\Delta_{\underline{\pi}}^{0}(y) \supseteq \Delta_{\underline{\pi}}^{1}(y) \supseteq \Delta_{\underline{\pi}}^{2}(y) \supseteq \ldots$ with corresponding sum formula. Although the first term of each of these filtrations $\bar{\Delta}_{\underline{\pi}}^{1}(y)$ is always the radical of the form on $\Delta_{\underline{\underline{\pi}}}(y)$, the other terms of the filtration will in general depend on the valuation $\nu$.

A particularly interesting case of our theory is the case where $W$ is of type $A_{n-1}$ and $\mathbb{k}=\overline{\mathbb{F}}_{p}$, since the decomposition numbers for $A_{\underline{\pi}}$ in this case are the decomposition numbers for the algebraic group $S l_{n}\left(\overline{\mathbb{F}}_{p}\right)$ around the Steinberg weight, by Riche and Williamson's recent work. In this case, our sum formula looks as follows

$$
\sum_{i>0}\left[\Delta_{\pi, \mathbb{F}_{p}}^{i}(y)\right]=\sum_{\beta>0, s_{\beta} y>y} \nu_{p}(\beta)\left[\Delta_{\pi, \overline{\mathbb{F}}_{p}}\left(s_{\beta} y\right)\right]
$$


where $\nu_{p}(\beta)$ is the canonical $p$-adic valuation, see Theorem 8.8 We give in the end of the paper an example to illustrate how to use this formula to obtain decomposition numbers.

There are several Jantzen type filtrations with associated sum formulas available in the literature, for example Andersen's filtrations for tilting modules. At present, we do not know if it makes sense to ask for possible relations between these filtrations and ours.

The paper is organized as follows. In section 2 we describe the basic facts of Soergel bimodules. In section 3 we explain the diagrammatical category $\mathcal{D}$ and the diagrammatical category $\mathcal{D}^{s t d}$, with an emphasis on the localization methods. Next, in section 4, we describe the cellular basis for $\mathcal{D}$, the light leaves basis. In section 5 we introduce the $L_{i}$ 's and prove that they are JM-elements. This relies heavily on the previous sections. We moreover show that they satisfy a separability condition over the fraction field. In section 6 we obtain via the JM-elements a first version of the determinant formula and then, in section 7, we get via Plaza's branching rule the Shapovalov type version of the determinant formula, mentioned above. In section 8 we construct the quasi-hereditary algebra $A_{\pi}$ and use it to deduce the sum formula from the determinant expression. Finally, in section 9 we give an application of our formula.

It is a pleasure to thank David Plaza for useful conversations related to this article. It is also a great pleasure to thank the anonymous referee for a long list of comments and suggestions that helped us improve the exposition of the article.

This paper is published in Journal of Algebra, but this version of the paper differs in some places from the published version. After the publication of the paper H. H. Andersen pointed several inaccuracies out to us, especially in the last section 9 where the $A_{2}$-calculations are done, and the differences from the published version of the paper are essentially due to the corresponding corrections. We thank H. H. Andersen for mentioning these inaccuracies.

\section{The category of Soergel bimodules}

In this section, we briefly explain the basic facts of the category of Soergel bimodules.

Let $(W, S)$ be a Coxeter system. Thus $W$ is the group generated by the finite set $S$ subject to the relations $(s t)^{m_{s t}}=1$ where the $m_{s t}$ 's are fixed numbers $m_{s t} \in\{1,2,3, \ldots, \infty\}$ satisfying $m_{s t}=m_{t s}$ and that $m_{s t}=1$ iff $s=t$. Here $m_{s t}=\infty$ means that the relation $(s t)^{m_{s t}}=1$ is omitted. We denote by $<$ the (Chevalley)-Bruhat order on $W$; we use the convention that $1 \in W$ is the smallest element with respect to $<$.

We shall make a careful distinction between elements $w$ of $W$ and expressions $\underline{w}$ in $S$, that is words of finite length in the alphabet $S$, including the empty word. We denote by $\exp _{s}$ the set of expressions in $S$ and by $\operatorname{rexp}_{s}$ the subset of $\exp _{s}$ consisting of reduced expressions in $S$. There is a canonical map $\exp _{s} \rightarrow W, \underline{w} \mapsto w$ which we use tacitly. For example, if $s \in S$ and $\underline{w}:=s s$ then $\underline{w} \mapsto w=1$.

Let $\mathbb{k}$ be a field. The setting of the theory of Soergel bimodules is a representation $\mathfrak{h}$ of $W$ over $\mathbb{k}$ which is reflection faithful in the sense of Soergel, see [31. Soergel used in loc. cit. an extension of a construction of Kac to show that a reflection faithful representation of $(W, S)$ exists when $\mathbb{k}=\mathbb{R}$. However, for infinite $W$ and for $\mathbb{k}$ of positive characteristic, it does not exist in general.

Let $\mathfrak{h}$ be a reflection faithful representation of $(W, S)$ and let $R:=\oplus_{m} S^{m}\left(\mathfrak{h}^{*}\right)$ be the symmetric algebra on $\mathfrak{h}^{*}$. The elements $f$ of $R$ are the polynomial functions in $\mathfrak{h}$. The $W$-action on $\mathfrak{h}$ induces a $W$-action on $\mathfrak{h}^{*}$, and so on $R$, and for $s \in S$ the corresponding subalgebra of $s$-invariants is denoted $R^{s}$, that is $R^{s}:=\{x \in R \mid s x=x\}$.

There is a natural $\mathbb{Z}$-grading on $R$ which is chosen such that the elements of $\mathfrak{h}^{*}$ are of degree two. The $W$-action on $R$ is degree preserving and so $R^{s}$ is a $\mathbb{Z}$-graded subalgebra of $R$.

When referring to 'grading', we always mean 'ZZ-grading'. In general, for a graded commutative ring $S$ and a graded $S$-module $M=\oplus_{i} M^{i}$ we let $M(k)$ denote the graded $S$-module obtained by shifting the degree, that is $M(k):=\oplus_{i} M(k)^{i}$ where $M(k)^{i}:=M^{i+k}$. 
For $s \in S$ we define the graded $R$-bimodule $B_{s}:=R \otimes_{R^{s}} R(1)$ and for $\underline{w}=s_{i_{1}} s_{i_{2}} \cdots s_{i_{k}} \in \exp _{s}$ we define the graded $R$-bimodule

$$
B_{\underline{w}}:=B_{s_{i_{1}}} \otimes_{R} B_{s_{i_{2}}} \otimes_{R} \cdots \otimes_{R} B_{s_{i_{k}}} .
$$

The action of $R$ on $B_{\underline{w}}$ is given by left and right multiplication. This is the Bott-Samelson bimodule associated with $\underline{w}$. The category of Bott-Samelson bimodules $\mathbb{B S B i m}$ is defined as the category whose objects are sums of shifts of Bott-Samelson bimodules and the category of Soergel bimodules $\mathbb{S B i m}$ is defined as the category whose objects are sums of shifts of summands of Bott-Samelson bimodules, where in both categories the morphisms are homogeneous $R$-bimodule homomorphisms. In other words, $\mathbb{S B i m}$ is the Karoubian envelope of $\mathbb{B S B i m}$.

Let $\mathcal{H}(W, S)$ be the Hecke algebra associated with $(W, S)$. It is the $\mathbb{Z}\left[q, q^{-1}\right]$-algebra with generators $\left\{H_{s} \mid s \in S\right\}$ subject to the relations $\left(H_{s}-q\right)\left(H_{s}+q^{-1}\right)=0$ and

$$
\underbrace{H_{s} H_{t} H_{s} \ldots}_{m_{s t} \text { factors }}=\underbrace{H_{t} H_{s} H_{t} \ldots}_{m_{s t} \text { factors }}
$$

For $w \in W$ represented by the reduced expression $w=s_{i_{1}} s_{i_{2}} \cdots s_{i_{k}}$ we set $H_{w}:=H_{i_{1}} H_{i_{2}} \cdots H_{i_{k}}$ which by Matsumoto's Theorem does not depend on the choice of reduced expression. Then $\left\{H_{w} \mid w \in W\right\}$ is a $\mathbb{Z}\left[q, q^{-1}\right]$-basis for $\mathcal{H}(W, S)$. Let $\left\{\underline{H}_{w} \mid w \in W\right\}$ be the Kazhdan-Lusztig basis for $\mathcal{H}(W, S)$ in the normalization used in [31] for example $\underline{H}_{s}=H_{s}+q$. The entries of the base change matrix between the two bases for $\mathcal{H}(W, S)$ are the Kazhdan-Lusztig polynomials, by definition.

Let $\langle\mathbb{S B i m}\rangle$ be the split Grothendieck group of $\mathbb{S B i m}$ and let $[M]$ denote the class in $\langle\mathbb{S B i m}\rangle$ of the object $M \in \mathbb{S B i m}$. We make $\langle\mathbb{S B i m}\rangle$ into a $\mathbb{Z}\left[q, q^{-1}\right]$-algebra by the rules $[M][N]:=\left[M \otimes_{R} N\right]$ and $q[M]:=M[-1]$.

The following important Theorem was shown in 31. It is known as Soergel's categorification Theorem.

Theorem 2.1. a) For each reduced expression $\underline{w} \in \operatorname{rexp}_{s}$ of $w \in W$ there is a unique indecomposable bimodule $B_{w}$ in $\mathbb{S B i m}$ that occurs as a summand of $B_{\underline{w}}$ (with multiplicity one) and does not occur as a summand of any $B_{y}$ such that $y \in \operatorname{rexp}_{s}$ and $y<\bar{w}$. The set $\left\{B_{w}(m) \mid w \in W, m \in \mathbb{Z}\right\}$ classifies the indecomposable bimodules in $\overline{\mathrm{SB}}$ Bim up to isomorphism.

b) There is a unique algebra homomorphism $F: \mathcal{H}(W, S) \rightarrow\langle\mathbb{S B i m}\rangle$ given by $\underline{H}_{s} \mapsto\left[B_{s}(1)\right]$. It is an isomorphism of $\mathbb{Z}\left[q, q^{-1}\right]$-algebras.

Soergel's conjecture from [31] states that if $\mathbb{k}=\mathbb{R}$, then $F\left(\underline{H}_{w}\right)=\left[B_{w}\right]$. It implies positivity properties for the Kazhdan-Lusztig polynomials and if $W$ is the Weyl group associated with a complex semisimple Lie algebra $\mathfrak{g}$, it also implies the Kazhdan-Lusztig conjectures for $\mathfrak{g}$. For Weyl groups it was shown by Soergel in [32] and for general Coxeter groups it was recently shown in a seminal work by Elias and Williamson, see [4].

\section{The diagrammatical categories $\mathcal{D}$ and $\mathcal{D}^{\text {std }}$}

Let $A_{\underline{w}}:=\operatorname{End}_{\mathbb{B S B i m}}\left(B_{\underline{w}}\right)$. It is shown in [5] that $\mathbb{B S B i m}$ is a cellular category, and in particular that $A_{\underline{w}}$ is a cellular algebra in the sense of Graham and Lehrer. This result is a starting point of our paper and we need to explain it in some detail.

The cellularity of $A_{\underline{w}}$ comes from the representation of $\mathbb{B S B i m}$ as a diagrammatical category $\mathcal{D}$. For general Coxeter systems $(W, S)$ this diagrammatical category $\mathcal{D}$ was constructed by Elias and Williamson in [5] although preliminary versions of $\mathcal{D}$ already appeared in [7] and [3]. One advantage of $\mathcal{D}$ over the bimodule category $\mathbb{B S B i m}$ is that it makes sense even for infinite $W$ in positive characteristic.

The setting for $\mathcal{D}$ is a realization $\mathfrak{h}$ of $(W, S)$, as introduced in section 3 of [ $[\mathbf{5}$.

Definition 3.1. A realization of $(W, S)$ over a commutative ring $\mathbb{k}$ is a free, finite rank module $\mathfrak{h}$ over $\mathbb{k}$ together with sets $\left\{\alpha_{s} \mid s \in S\right\} \subset \mathfrak{h}^{*}$ and $\left\{\alpha_{s}^{\vee} \mid s \in S\right\} \subset \mathfrak{h}$ such that $\left\langle\alpha_{s}^{\vee}, \alpha_{s}\right\rangle=2$ and such that the rule $s(v):=v-\left\langle v, \alpha_{s}\right\rangle \alpha_{s}^{\vee}$ defines a representation of $W$ in $\mathfrak{h}$. Furthermore, a technical 'balancedness' condition should be verified. 
We shall always assume that Demazure surjectivity holds.

Definition 3.2. A realization $\mathfrak{h}$ of $(W, S)$ over $\mathbb{k}$ is said to be Demazure surjective if for all $s \in S$ the evaluation maps $\alpha_{s}: \mathfrak{h} \rightarrow \mathbb{k}$ and $\alpha_{s}^{\vee}: \mathfrak{h}^{*} \rightarrow \mathbb{k}$ are surjective.

Given a realization of $\mathfrak{h}$ of $(W, S)$, the graded commutative ring $R$ is defined as in the bimodule case. Let us now explain the various other ingredients of $\mathcal{D}$, as introduced in [5].

Definition 3.3. A diagram for $(W, S)$ (or simply a diagram when confusion is not possible) is a finite diagram on a strip $\mathbb{R} \times[0,1]$. The arcs are decorated with elements of $S$. The vertices are the points where the arcs end or meet. The intersection points between the arcs and the upper (lower) border of the strip $\mathbb{R} \times\{1\}(\mathbb{R} \times\{0\})$ are called boundary point and are not vertices. They define sequences of elements of $S$, called the top sequence and bottom sequence of the diagram. The arcs all end in vertices or boundary points. Loops are allowed. The regions defined by the arcs may be decorated by homogeneous elements of $R$. All diagrams are considered up to isotopy.

The diagram is called standard if the only vertices are $2 m_{\text {st }}$-valent, with incident lines of alternating decorations $s, t, \ldots, s, t$.

The diagram is called a Soergel diagram if each vertex is either 0-valent, that is an endpoint of an arc, or 3-valent with the three incident arcs of the same colour, or $2 m_{s t}$-valent, with incident arcs of alternating decorations $s, t, \ldots, s, t$. The degree of a Soergel diagram is the sum of the degrees of all its vertices and polynomials where a 0 -valent vertex has degree 1, a 3-valent vertex has degree -1 and a $2 m_{\text {st }}$-valent vertex has degree 0 .

When we draw diagrams, we identify $S$ with a set of physical colours and indicate the decorations of the lines by using those colours. Below is an example of a Soergel diagram of degree 5 with $S:=\{$ red,blue,green $\}$ and $m_{\text {red,blue }}=3, m_{\text {red,green }}=2$ and $m_{\text {blue, green }}=2$.

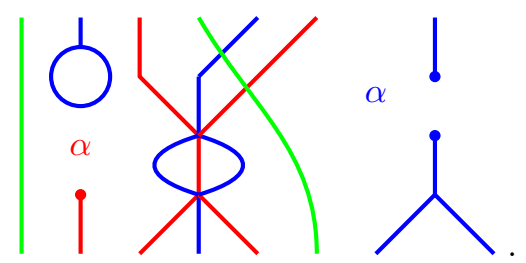

Standard diagrams are drawn using dashed lines. Below is an example, using the same $S$ and $m$ 's as before.

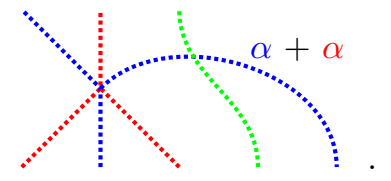

We can now give the definition of the category $\mathcal{D}$.

Definition 3.4. Let $\mathbb{k}$ be as before. Then $\mathcal{D}$ is the $\mathbb{k}$-linear monoidal category whose objects are the elements of $\exp _{s}$. For $\underline{w}, \underline{z} \in \exp _{s}$, the morphism space $\operatorname{Hom}_{\mathcal{D}}(\underline{w}, \underline{z})$ is the free $\mathbb{k}$-module spanned by Soergel diagrams with bottom sequence $\underline{w}$ and top sequence $\underline{z}$ modulo a number of relations, that we explain below. The composition $g \circ f$ of a morphism $f \in \operatorname{Hom}_{\mathcal{D}}(\underline{w}, \underline{z})$ and a morphism $g \in \operatorname{Hom}_{\mathcal{D}}(\underline{z}, \underline{x})$ is given by vertical concatenation, with $g$ on top of $f$.

The monoidal structure is given by horizontal concatenation of expressions.

Let us now explain the relations between the diagrams in $\operatorname{Hom}_{\mathcal{D}}(\underline{w}, \underline{z})$. The isotopy relation has already been mentioned. The other relations are divided into the polynomial relations, the one-colour, two-colour, three-colour relations and the cyclicity relation. They are all local, involving a small part of the diagram and leaving the rest unchanged. The polynomial and one-colour relations are the following ones (for the colour blue):

$$
\begin{gathered}
l=\alpha \\
f|=| s f+\underset{b}{\grave{i}}
\end{gathered}
$$




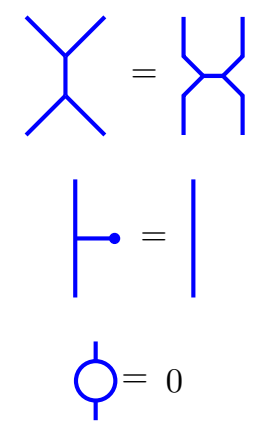

where $\partial: R \rightarrow R$ is the Demazure operator given by

$$
\partial(f):=\frac{f-s(f)}{\alpha}
$$

We do not explain the remaining relations, but instead refer the reader to Definition 5.2 of [5].

Let us now suppose that $\mathbb{k}$ is a field and that $\mathfrak{h}$ is a Soergel realization of $(W, S)$, in the sense of [5], meaning that $\mathfrak{h}$ is a faithful representation of $W$ such that Theorem 2.1 holds with respect to $\mathfrak{h}$. Then by [5] there is an equivalence of categories $\mathcal{F}: \mathcal{D} \rightarrow \mathbb{B S B i m}$, defined on objects by $\mathcal{F}(\underline{w}):=B_{\underline{w}}$, where $B_{\emptyset}:=R$. On one-colour morphisms $\mathcal{F}$ is defined as follows

$$
\mid \begin{aligned}
& \mapsto \mapsto \quad f \otimes g \mapsto f g \\
& \mapsto \mapsto \frac{1}{2}(\alpha \otimes 1+1 \otimes \alpha) \\
& \mapsto \mapsto 1 \otimes g \otimes 1 \mapsto \partial g \otimes 1
\end{aligned}
$$

(Here, if $\mathbb{k}$ is of characteristic 2 , one should replace $\frac{1}{2}(\alpha \otimes 1+1 \otimes \alpha)$ in (3.9) by $\Delta_{s}:=\delta \otimes 1-1 \otimes s(\delta)$ where $\delta$ is defined by $\partial(\delta)=1$; it exists because of Demazure surjectivity). On polynomials, $\mathcal{F}$ is defined by mapping $f \in R$ to multiplication by $f$ in the slot of $B_{\underline{w}}$ associated with the region associated with $f$. We do not here explain the image of $\mathcal{F}$ on diagrams involving more colours but refer the reader to Definition 5.12 of [5].

This equivalence of categories allows us to answer questions related to $\mathbb{B S B i m}$ in $\mathcal{D}$.

We now explain the diagrammatic category $\mathcal{D}^{s t d}$. It is an auxiliary category with a particularly simple structure that we shall rely on for our main results.

Definition 3.5. Let $\mathbb{k}$ be a commutative ring. Then $\mathcal{D}^{\text {std }}$ is the additive $\mathbb{k}$-linear monoidal category whose objects are direct sums of elements of $\exp _{s}$. For $\underline{w}, \underline{z} \in \exp _{s}$ the morphism space $\operatorname{Hom}_{\mathcal{D}^{\text {std }}}(\underline{w}, \underline{z})$ is the $\mathbb{k}$-module spanned by standard diagrams with bottom sequence $\underline{w}$ and top sequence $\underline{z}$ modulo a number of relations, that we explain shortly, and for direct sums of such objects we use the linear extension. The composition of morphisms and the monoidal structure on $\mathcal{D}^{\text {std }}$ are defined as in $\mathcal{D}$.

Let us briefly explain the relations. One should think of them as the relations in $\mathcal{D}$ with the 'lower terms' deleted. More precisely, the one-colour relations are the following

$$
f=s f
$$




$$
\begin{aligned}
& y=1 \\
& \vartheta^{\ddots} \cdot{ }^{\vdots}
\end{aligned}
$$

The two-colour relations are as follows (in the cases $m=2, m=3$ )

$$
y_{0}=\frac{b}{y}=
$$

which implies that the $2 m$-valent vertices are idempotents in $\mathcal{D}^{s t d}$. For an explanation of the remaining relations of $\mathcal{D}^{s t d}$, we refer the reader to section 4 of $[5]$.

An important remark is that $\mathcal{D}^{\text {std }}$ is a simpler category than $\mathcal{D}$. In fact we have that

$$
\operatorname{Hom}_{\mathcal{D}^{s t d}}(\underline{x}, \underline{y})= \begin{cases}\mathbb{k} & \text { if } x=y \\ 0 & \text { otherwise }\end{cases}
$$

see the remarks following Theorem 4.8 of [5] and [6]. Indeed, for $\underline{y}, \underline{y^{\prime}} \in \exp _{s}$ with $y=y^{\prime}$ there is a unique diagram $\operatorname{Std}\left(\underline{y}, \underline{y^{\prime}}\right)$ in $\mathcal{D}^{s t d}$ from $\underline{y}$ to $\underline{y^{\prime}}$ and the basis for $\bar{H}_{\mathcal{D}^{s t d}}\left(\underline{y}, \underline{y^{\prime}}\right)$ is given by that diagram.

Let $Q:=Q(R)$ be the quotient field of $R$. We are interested in the corresponding category $\mathcal{D}_{Q}$, in which elements of $Q$ are allowed in the regions of the diagrams. There is a canonical localization functor $\mathcal{D} \rightarrow \mathcal{D}_{Q}$ which is injective on the Hom-spaces, since $\operatorname{Hom}_{\mathcal{D}}(\underline{w}, y)$ is free over $R$ (as we shall see shortly). The following Theorem, see Definition 5.15 and Theorem $5.1 \overline{6}$ of [ $[$, gives a diagrammatical description of the Karoubian envelope of $\mathcal{D}_{Q}$.

Theorem 3.6. $\operatorname{Kar}\left(\mathcal{D}_{Q}\right)$ is the additive $Q$-linear monoidal category whose objects are direct sums of expressions $\underline{w}$ over $S$ as before, but where each letter $s \in S$ may now be a normal index or a reflection index. The morphism of the normal indices are drawn normally, whereas the morphisms of the reflection indices are drawn using dashed arcs. The morphisms in $\operatorname{Hom}_{K} \operatorname{rar}_{\left(\mathcal{D}_{Q}\right)}(\underline{w}, \underline{y})$ are spanned over $Q$ by Soergel diagrams from $\underline{w}$ to $y$ allowing normal arcs and dashed arcs where bivalent vertices, involving the same colour, like the following ones, are allowed:

$$
\iota=\mid \pi=
$$

The relations in $\operatorname{Kar}\left(\mathcal{D}_{Q}\right)$ involving undashed morphisms are the same as in $\mathcal{D}_{Q}$ and the relations involving dashed morphisms are those of $\mathcal{D}_{Q}^{\text {std }}$. The relations involving bivalent vertices are the following ones (in the case of the colour blue)

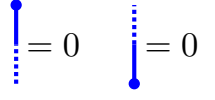

$$
\begin{aligned}
& \left|=\frac{1}{\alpha}+\frac{1}{\alpha}\right| \\
& =\alpha \\
& \because=\frac{1}{\alpha} \bigcap \\
& =\frac{1}{\alpha} \bigcup
\end{aligned}
$$


There is a simple description of $\operatorname{Kar}\left(\mathcal{D}_{Q}\right)$. Indeed, let $D$ be a diagram in $\operatorname{Kar}\left(\mathcal{D}_{Q}\right)$. Then applying (3.19) to all the top and bottom arcs of $D$ and using the relations of $\operatorname{Kar}\left(\mathcal{D}_{Q}\right)$, together with the relation (3.16), one gets that $D$ becomes a linear combination of diagrams $S\left(\underline{y}, \underline{y^{\prime}}\right)$ in which each $S\left(\underline{y}, \underline{y^{\prime}}\right)$ is of the form $S t d\left(\underline{y}, \underline{y^{\prime}}\right)$ but with the top (dashed) arcs extended to the upper border via! and

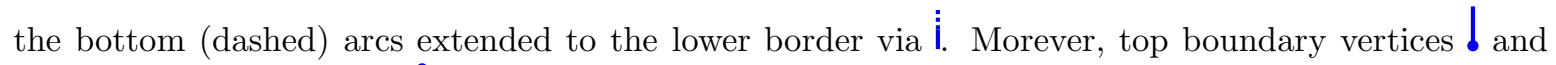
bottom boundary vertices $i$ are allowed. The details of this description are given in section 5 of [ $[$.

\section{Cellularity of $\mathcal{D}$.}

Let us now return to the category $\mathcal{D}$. Elias and Williamson showed in Proposition 6.23 of [5] that it is a cellular category, in the the sense of [33]. In particular, for any $\underline{w} \in \exp _{s}$ we have that $A_{\underline{w}}=\operatorname{End}_{\mathcal{D}}\left(B_{\underline{w}}\right)$ is a cellular algebra. In this section we explain the various ingredients of the cellular structure of $\overline{\mathcal{D}}$ according to [5]. The cellular basis itself is a diagrammatical version of Libedinsky's light leaves basis, see 16 .

Let us first recall the original definition of a cellular algebra, as formulated by Graham and Lehrer in 8 .

Definition 4.1. Let $A$ be a finite dimensional algebra over a commutative ring $\mathbb{k}$. Then a cellular basis for $A$ is a triple $(\Lambda$, Tab, $C)$ where $\Lambda$ is a poset, Tab is a function on $\Lambda$ with values in finite sets and $C: \coprod_{\lambda \in \Lambda} \operatorname{Tab}(\lambda) \times \operatorname{Tab}(\lambda) \rightarrow A$ is an injection such that

$$
\left\{C_{s t}^{\lambda} \mid s, t \in \operatorname{Tab}(\lambda), \lambda \in \Lambda\right\}
$$

is a $\mathbb{k}$-basis for $A$ : the cellular basis for $A$. The rule $\left(C_{s t}^{\lambda}\right)^{*}:=C_{t s}^{\lambda}$ defines a $\mathbb{k}$-linear antihomomorphism of $A$ and, finally, the structure constants for $A$ with respect to $\left\{C_{s t}^{\lambda}\right\}$ satisfy the following condition with respect to the partial order: for all $a \in A$ we have

$$
a C_{s t}^{\lambda}=\sum_{u \in \operatorname{Tab}(\lambda)} r_{u s a} C_{u t}^{\lambda}+\text { lower terms }
$$

where lower terms means a linear combination of $C_{a b}^{\mu}$ where $\mu<\lambda$ and where $r_{u s a} \in \mathbb{k}$.

Let us now explain the various ingredients of the cellular basis for $A_{\underline{w}}$, given in [5]. For $\mathbb{k}$ we choose $R$ itself. Suppose that $\underline{w}=s_{i_{1}} s_{i_{2}} \cdots s_{i_{k}} \in \mathbf{e x p}_{s}$. We then define a subexpression of $\underline{w}$ to be a sequence $\mathbf{e}=\left(e_{1}, e_{2}, \ldots, e_{k}\right) \in\{0,1\}^{k}$. For each such subexpression e we define $\underline{w}^{\mathbf{e}}:=s_{i_{1}}^{e_{1}} s_{i_{2}}^{e_{2}} \cdots s_{i_{k}}^{e_{k}} \in \mathbf{e x p}_{s}$ and we define $w^{\mathbf{e}} \in W$ as the corresponding group element. If $w^{\mathbf{e}}=y$ then $\mathbf{e}$ is said to express $y$ and in this case we write $y \leq \underline{w}$. Note that if $\underline{w} \in \operatorname{rexp}_{s}$, then we have that $y \leq \underline{w}$ iff $y \leq w$.

Let $\underline{w}^{\mathbf{e}}=s_{i_{1}}^{e_{1}} s_{i_{2}}^{e_{2}} \cdots s_{i_{k}}^{e_{k}}$. For $\Lambda$ we choose the subset of $W$ given by $\left\{w^{\mathbf{e}} \mid \mathbf{e}\right.$ is a subexpression of $\left.\underline{w}\right\}$ with the poset structure being induced from the Bruhat order on $W$. For the Tab function we choose

$$
\operatorname{Tab}(y):=\left\{\mathbf{e} \in\{0,1\}^{k} \mid \mathbf{e} \text { is a subexpression of } \underline{w} \text { expressing } y\right\} .
$$

We are now only left with the definition of the cellular basis itself. We need some combinatorial concepts related to subexpressions. For $\underline{w}=s_{i_{1}} \cdots s_{i_{j}} \cdots s_{i_{k}}$ we define $\underline{w} \leq j=s_{i_{1}} \cdots s_{i_{j}} \in \mathbf{e x p}_{s}$. Any subexpression e of $\underline{w}$ defines a sequence $\left(\underline{w}_{0}, \underline{w}_{1}, \ldots, \underline{w}_{k}\right)$ in $\exp _{s}$ via $\underline{w}_{0}=1$ and recursively

$$
\underline{w}_{j}= \begin{cases}\underline{w}_{j-1} s_{j} & \text { if } e_{j}=1 \\ \underline{w}_{j-1} & \text { otherwise. }\end{cases}
$$

This gives rise to a series of symbols $T=\left(t_{1}, t_{2}, \ldots, t_{k}\right) \in\{U, D\}^{k}$ ( $U=$ up, $D=$ down) defined as follows

$$
t_{j}= \begin{cases}U & \text { if } w_{j-1} s_{j}>w_{j-1} \\ D & \text { otherwise }\end{cases}
$$

In particular, we always have that $t_{1}=U$. We merge the sequences $T$ and e into one sequence $M=\left(m_{1}, m_{2}, \ldots, m_{k}\right)$ by concatenating the symbols, that is $M_{j}:=t_{j} e_{j}$. 
With this notation at hand, we construct a series of morphism $\mathbb{L}_{\underline{w}, \mathbf{e}, \leq j} \in \operatorname{Hom}_{\mathcal{D}}\left(B_{\underline{w} \leq j}, B_{\underline{w}_{j}}\right)$ as follows. We first let $\mathbb{L}_{\underline{w}, \mathbf{e}, \leq 0}$ be the empty diagram. Suppose recursively that $\alpha:=\mathbb{L}_{\underline{w}, \mathbf{e}, \leq j-1}$ has already been constructed. Then $\mathbb{L L}_{\underline{w}, \mathbf{e}, \leq j}$ is obtained from $\alpha$ by first adding on the right a vertical arc of colour $i_{j}$. This arc is then further manipulated in a way that depends on the value of $M_{j}$. The rules are as follows.

- If $M_{j}=U 0$, then the new arc is terminated with a dot.

- If $M_{j}=U 1$, then the new arc is continued to the top.

- If $M_{j}=D 0$, then $s_{i_{j}}$ is in the right descent set of $w_{k-1}$ and one applies a series of $m$-valent vertices to $\alpha$ such that the result has an arc of colour $i_{j}$ to the right of $\alpha$. Finally a trivalent vertex of colour $i_{j}$ is applied to the final two strands.

- If $M_{j}=D 1$, then we proceed as in case $D 0$ but finish with a cap of colour $i_{j}$.

The four cases have the following diagrammatical representations:

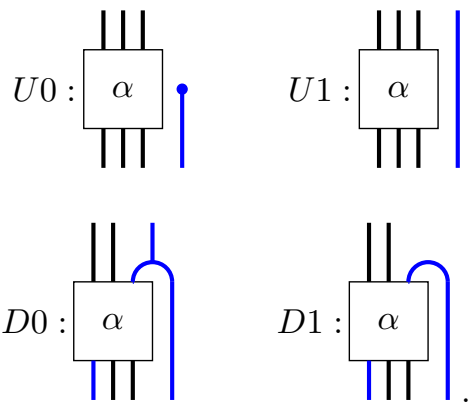

Finally we set $\mathbb{L L}_{\underline{w}, \mathbf{e}}:=\mathbb{L}_{\underline{w}, \mathbf{e}, \leq k}$ : this is the light leaves morphism associated with $\mathbf{e}$. It is a diagrammatical version of the bimodule homomorphism introduced by Libedinsky, see [16].

For all $\mathbf{e} \in \operatorname{Tab}(y)$ our chosen $\mathbb{L L}_{\underline{w}, \mathbf{e}}$ belongs to $\operatorname{Hom}_{\mathcal{D}}\left(B_{\underline{w}}, B_{\underline{w} \mathbf{e}}\right)$ where $\underline{w}^{\mathbf{e}}=y$. We choose a fixed expression $y$ for $y$ and fix for all appearing $\underline{w}^{\mathbf{e}}$ a set of braid moves transforming $\underline{w}^{\mathbf{e}}$ to $y$. We then modify the $\overline{\mathbb{L}} \mathbb{L}_{\underline{w}, \text { e }}$ by multiplying with the corresponding set of $m$-valent vertices. In this way, all chosen $\mathbb{L}_{\underline{w}, \mathbf{e}}$ now belong to the same $\operatorname{Hom}_{\mathcal{D}}\left(B_{\underline{w}}, B_{\underline{y}}\right)$.

It should be noted that although the $\mathbb{L L}_{\underline{w}}$, e's depend heavily on the choices of $m$-valent vertices along the way, all choices will do for our results.

For $\mathbf{e}, \mathbf{e}_{1} \in \operatorname{Tab}(y)$ we define $\beta:=\mathbb{L L}_{\underline{w}, \mathbf{e}}$ and $\beta_{1}:=\mathbb{L}_{\underline{w}}, \mathbf{e}_{1}$. Let $\beta_{1}^{*} \in \operatorname{Hom}_{\mathcal{D}}\left(B_{\underline{y}}, B_{\underline{w}}\right)$ be the morphism obtained from $\beta_{1}$ by reflection along a horizontal axis. We then define the last ingredient $C$ of the cellular basis as $C_{\mathbf{e}_{1}}^{y}=\mathbb{L} \mathbb{L}_{\mathbf{e}, \mathbf{e}_{1}, y}:=\beta_{1}^{*} \beta \in A_{\underline{w}}$. Let us illustrate the light leaves basis in a couple of simple examples. Let first $W$ be of type $A_{1}$, that is $S=\{s\}$. Then $W$ has just two elements $\{1, s\}$ and all diagrams are one-coloured, of colour blue. Let us take $\underline{w}:=s s s$ representing $s$. Then we have

$$
\begin{gathered}
\operatorname{Tab}(1)=\{(0,0,0),(1,1,0),(0,1,1),(1,0,1)\} \\
\operatorname{Tab}(s)=\{(0,0,1),(1,1,1),(0,1,0),(1,0,0)\} .
\end{gathered}
$$

The corresponding symbols $M$ are

$$
\begin{aligned}
& \{(U 0, U 0, U 0),(U 1, D 1, U 0),(U 0, U 1, D 1),(U 1, D 0, D 1)\} \\
& \{(U 0, U 0, U 1),(U 1, D 1, U 1),(U 0, U 1, D 0),(U 1, D 0, D 0)\}
\end{aligned}
$$

and so the corresponding light leaves diagrams in $\operatorname{Tab}(1)$ are

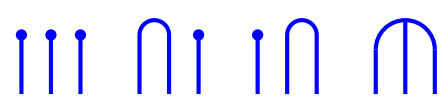

and in $\operatorname{Tab}(s)$ they are

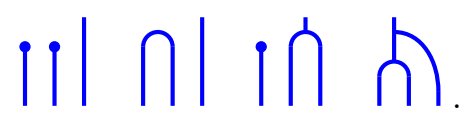


Thus the cellular basis for $\operatorname{End}_{\mathcal{D}}\left(B_{\underline{w}}\right)$ has 32 elements. Here are four of them corresponding to $\operatorname{Tab}(1) \times$ $\operatorname{Tab}(1)$

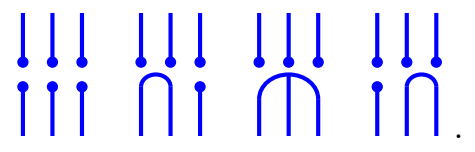

It should be mentioned that in [17] Libedinsky gives a non-recursive description of $\operatorname{End}_{\mathcal{D}}\left(B_{\underline{w}}\right)$, for all $\underline{w} \in \operatorname{rexp}_{s}$, but only in the $A_{1}$ and $\tilde{A}_{1}$-cases.

Let us next consider type $A_{2}$, that is $S:=\{\alpha, \beta\}$ with $m_{\alpha \beta}=3$. Let us take $s_{1}:=\alpha$ and $s_{2}:=\beta$ and let $\underline{w}:=s_{1} s_{2} s_{1}$. Then $\operatorname{Tab}(1)=\{(0,0,0),(1,0,1)\}$ with symbols $\{(U 0, U 0, U 0),(U 1, U 0, D 1)\}$ and the corresponding light leaves morphisms are

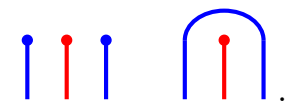

We have $\operatorname{Tab}\left(s_{1}\right)=\{(1,0,0),(0,0,1)\}$ with symbols $\{(U 1, U 0, D 0),(U 0, U 0, U 1)\}$ and light leaves morphisms

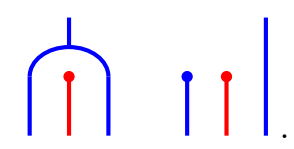

We have $\operatorname{Tab}\left(s_{1} s_{2}\right)=\{(1,1,0)\}$ with symbols $\{(U 1, U 1, U 0)\}$ and light leaf

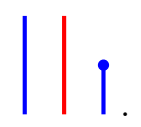

Let us finally mention $\operatorname{Tab}\left(s_{1} s_{2} s_{1}\right)=\{(1,1,1)\}$ that corresponds to the identity map

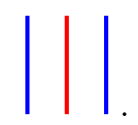

\section{Jucys-Murphy elements}

Let us fix an expression $\underline{w}=s_{i_{1}} s_{i_{2}} \cdots s_{i_{k}} \in \exp _{s}$. In this section we consider for $i \in\{1,2, \ldots, k\}$ the diagram $L_{j} \in A_{\underline{w}}$ given by

$$
\begin{aligned}
& \text { j'th position }
\end{aligned}
$$

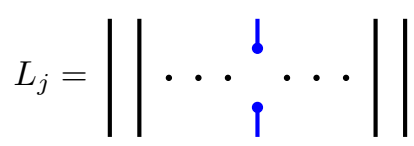

where the first colour black refers to $i_{1}$, the second colour black refers to $i_{2}$ and so on, except that the blue colour refers to $i_{j}$. In this section we study the left multiplication by $L_{j}$ on the light leaves basis $\left\{\mathbb{L} \mathbb{L}_{\mathbf{e}, \mathbf{e}_{1}, y}\right\}$. It is interesting to note that the elements $L_{j}$ already appear, somewhat hidden, in Elias and Williamson's proof of Soergel's conjecture, see [4. Indeed, suppose that $\underline{w}=s_{i_{1}} s_{i_{2}} \cdots s_{i_{k}}$ and set $\overline{B_{\underline{w}}}:=B_{\underline{w}} \otimes_{R} \mathbb{R}$ where $\mathbb{R}$ is made into an $R$ algebra by mapping elements of positive degree to zero. Then the Lefschetz element $\rho$ introduced in [4], acting on $\overline{B_{\underline{w}}}$, satisfies by Lemma 3.4 of [4] the formula

$$
\rho(-)=\sum_{j=1}^{k}\left(s_{i_{j-1}} \ldots s_{i_{1}} \rho\right)\left(\alpha_{i_{j}}^{\vee}\right) \chi_{j} \circ \phi_{j} .
$$

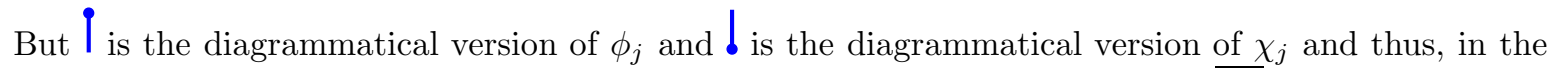
case where $\underline{w} \in \operatorname{rexp}_{s}$ we have that $\rho$ acts in the Bott-Samelson bimodule $\overline{B_{\underline{w}}}$, via an $\mathbb{R}^{+}$-linear combination of our $L_{j}$ 's. The main result of this section is that the set $\left\{L_{j} \mid \bar{j}=1, \ldots, k\right\}$ verifies the axiomatic condition, introduced by Mathas in [21, for being a set of JM-elements for $A_{\underline{w}}$ with 
respect to the light leaves basis. On the other hand, the results of 4 rely on positivity properties for $\rho$ over the real field $\mathbb{R}$. From this point of view, the formula (5.2) may indicate a connection between Lefschetz elements and JM-elements that could hold not only for Soergel bimodules, but also for other graded cellular algebras. It would be interesting to investigate this possible connection.

Let us recall Mathas' axiomatic condition for JM-elements.

Definition 5.1. Let $A$ be a cellular algebra over the commutative ring $\mathbb{k}$ with datum $(\Lambda$, Tab, $C)$. For each $\lambda \in \Lambda$, suppose that $\operatorname{Tab}(\lambda)$ is endowed with a poset structure with order relation $<$ (depending on $\lambda$ ). Let $\mathbf{L}=\left\{L_{1}, L_{2}, \ldots, L_{k}\right\}$ be a commutative family of elements of $A$, such that $L_{i}^{*}=L_{i}$ for all $i$. Suppose that there is a function $c_{a}:\{1,2, \ldots, k\} \rightarrow \mathbb{k}$ for each $a \in \operatorname{Tab}(\lambda)$. Then $\mathbf{L}$ is said to be $a$ family of JM-elements for $A$ with content functions $\left\{c_{a}\right\}$ if for all $i$ we have that

$$
L_{i} C_{a b}^{\lambda}=c_{a}(i) C_{a b}^{\lambda}+\text { lower terms }
$$

where lower terms means a linear combination of elements from $\left\{C_{a_{1} b}^{\lambda} \mid a_{1}<a\right\} \cup\left\{C_{a_{2} b_{2}}^{\mu} \mid \mu<\lambda\right\}$.

Let us now explain how our cellular algebra $A_{\underline{w}}$ fits into the framework of Definition 5.1 For the partial order on $\operatorname{Tab}(y)$ we use the path dominance order $\preceq$ introduced by Elias and Williamson. Let us explain it. Recall that a subexpression e of $\underline{w}$ defines a sequence $\left(\underline{w}_{1}, \underline{w}_{2}, \ldots, \underline{w}_{k}\right)$ in $\exp _{s}$ and hence also a sequence $\left(w_{1}, w_{2}, \ldots, w_{k}\right)$ of elements in $W$. If $\mathbf{f}$ is another subexpression of $\underline{w}$ with corresponding sequence $\left(v_{1}, v_{2}, \ldots, v_{k}\right)$ in $W$ then we say that $\mathbf{e} \preceq \mathbf{f}$ if $w_{i} \leq v_{i}$ for all $i$. If $\mathbf{e} \preceq \mathbf{f}$ we also say $\mathbb{L}_{\underline{w}, \mathbf{e}} \preceq \mathbb{L} \mathbb{L}_{\underline{w}, \mathbf{f}}$.

Let us consider the diagrams of (4.7). Let us denote them $D 1, D 2, D 3, D 4$ from left to right and let similarly the diagrams of (4.8) be denoted $E 1, E 2, E 3, E 4$. Then these diagrams are related via path dominance $\preceq$ as follows
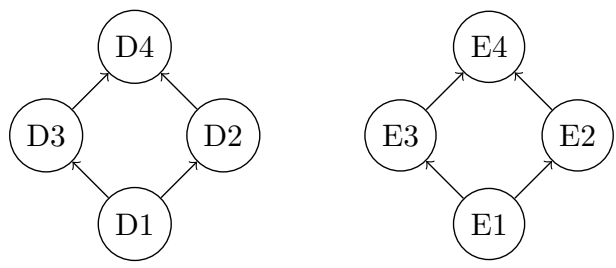

with $D 1$ and $E 1$ being the smallest and so on.

Recall that $\underline{w}=s_{i_{1}} s_{i_{2}} \cdots s_{i_{k}}$ is our fixed expression for $w$. Suppose that $\mathbf{e}=\left(e_{1}, e_{2}, \ldots, e_{k}\right) \in$ $\operatorname{Tab}(y)$ and that $T$ is the $a^{\prime}$ th coordinate of the string of symbols given by e, that is $T \in\{U 0, U 1, D 0, D 1\}$. We then define for $a=1,2, \ldots, k$ our candidate for the content function $c_{\mathbf{e}}:\{1,2, \ldots, k\} \rightarrow \mathbb{k}=R$ as follows

$$
c_{\mathbf{e}}(a):= \begin{cases}0 & \text { if } T=U 1, D 1 \\ s_{i_{1}}^{e_{1}} \ldots s_{i_{a-1}}^{e_{a-1}} \alpha_{i_{a}} & \text { if } T=U 0, D 0 .\end{cases}
$$

We are now in position to state and prove the promised Theorem. The proof uses Proposition 6.6 of [5] which is a main ingredient for showing the linear independence of the light leaves basis.

Theorem 5.2. The set $\mathbf{L}:=\left\{L_{1}, \ldots, L_{k}\right\}$ defined in (5.1) is a set of JM-elements for $A_{\underline{w}}$ with respect to the light leaves basis and path dominance order $\preceq$.

Proof: Clearly the $L_{i}$ 's commute and satisfy $L_{i}^{*}=L_{i}$ and hence we only need verify the lower triangularity condition (5.3). Recall that we have fixed a target object $\underline{y}=s_{j_{1}} \cdots s_{j_{l}} \in \mathbf{r e x p}_{s}$ for all light leaves morphisms in $\left\{\mathbb{L}_{\underline{w}, \mathbf{e}} \mid \mathbf{e} \in \operatorname{Tab}(y)\right\}$. Recall also the localization functor $\mathcal{D} \rightarrow \mathcal{D}_{Q}$; it induces a faithful functor $\mathcal{D} \rightarrow \operatorname{Kar}\left(\overline{\mathcal{D}}_{Q}\right)$. Using the description of $\operatorname{Kar}\left(\mathcal{D}_{Q}\right)$ given in the last paragraph of section 3 we have an expansion

$$
\mathbb{L L}_{\underline{w}, \mathbf{e}}=\sum_{\substack{\mathbf{f}, \mathbf{f}_{1} \\ \mathbf{f} \underline{\mathbf{e}}}} a_{\mathbf{f}, \mathbf{f}_{1}} S\left(\underline{w}^{\mathbf{f}}, \underline{y}^{\mathbf{f}_{1}}\right)
$$

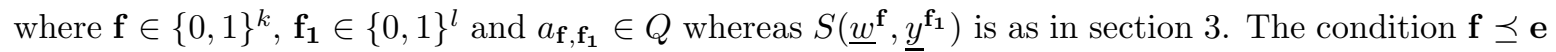
comes from the fact that the target object of $y^{\mathbf{f}_{1}}$ of $S\left(\underline{w}^{\mathbf{f}}, \underline{y}^{\mathbf{f}_{1}}\right)$ is a subexpression of $y$ and $y=w^{\mathbf{e}}$. The coefficient $a_{\mathbf{e},(1, \ldots, 1)}$ is calculated in [5] and is shown to be nonzero. 
We now get that

$$
\mathbb{L L}_{\underline{w}, \mathbf{e}} L_{i}=\sum_{\substack{\mathbf{f}, \mathbf{f}_{1} \\ \mathbf{f} \preceq \mathbf{e}}} a_{\mathbf{f}, \mathbf{f}_{1}} S\left(\underline{w}^{\mathbf{f}}, \underline{y}^{\mathbf{f}_{1}}\right) L_{i} .
$$

On the other hand, for all $\mathbf{f}$ and $\mathbf{f}_{\mathbf{1}}$ we have that

$$
S\left(\underline{w}^{\mathbf{f}}, \underline{y}^{\mathbf{f}_{1}}\right) L_{i}= \begin{cases}s_{j_{1}}^{f_{1}} s_{j_{2}}^{f_{2}} \ldots s_{j_{i-1}}^{f_{i-1}} \alpha_{i} S\left(\underline{w}^{\mathbf{f}}, \underline{y}^{\mathbf{f}_{1}}\right) & \text { if } f_{i}=0 \\ 0 & \text { if } f_{i}=1\end{cases}
$$

Indeed if $f_{i}=0$ then $S\left(\underline{w}^{\mathbf{f}}, \underline{y}^{\mathbf{f}_{1}}\right)$ has a bottom boundary dot $\uparrow$ at the $i$ 'th position and so the multiplication by $L_{i}$ gives rise to the scalar $\alpha_{i}$ which is pulled to the left whereas if $f_{i}=1$ then $S\left(\underline{w}^{\mathbf{f}}, \underline{y}^{\mathbf{f}_{\mathbf{f}}}\right)$ has a bottom $\dot{I}$ at the $i$ 'th position which is mapped to zero under the multiplication with $L_{i}$. We conclude from this that

$$
\mathbb{L}_{\underline{w}, \mathbf{e}} L_{i} \in \operatorname{span}_{Q}\left\{S\left(\underline{w}^{\mathbf{f}}, \underline{y}^{\mathbf{f}_{\mathbf{1}}}\right) \mid \mathbf{f} \preceq \mathbf{e}\right\} .
$$

Let us now fix an $\mathbf{e}_{0} \in \operatorname{Tab}(y)$ and consider $L_{i} \mathbb{L L}_{\mathbf{e}, \mathbf{e}_{0}, y} \in A_{\underline{w}}$. Using (5.6) and (5.9) we have that

$$
L_{i} \mathbb{L} \mathbb{L}_{\mathbf{e}, \mathbf{e}_{0}, y}=\left(\mathbb{L}_{\mathbb{L}_{w}, \mathbf{e}} L_{i}\right)^{*} \mathbb{L} \mathbb{L}_{\underline{w}, \mathbf{e}_{0}} \in \operatorname{span}_{Q}\left\{S\left(\underline{w}^{\mathbf{f}}, \underline{w}^{\mathbf{f}_{1}}\right) \mid \mathbf{f}, \mathbf{f}_{1} \preceq \mathbf{e}\right\} .
$$

Let us now consider the $R$-expansion of $L_{i} \mathbb{L L}_{\mathbf{e}, \mathbf{e}_{0}, y}$ in terms of the cellular basis:

$$
L_{i} \mathbb{L} \mathbb{L}_{\mathbf{e}, \mathbf{e}_{0}, y}=\sum_{\mathbf{e}_{1} \in \operatorname{Tab}(y)} a_{\mathbf{e}_{1}} \mathbb{L} \mathbb{L}_{\mathbf{e}_{1}, \mathbf{e}_{0}, y}+\text { lower terms. }
$$

We must prove that for all $\mathbf{e}_{1}$ appearing in (5.11) with $a_{\mathbf{e}_{1}} \neq 0$ we have that $\mathbf{e}_{1} \preceq \mathbf{e}$. Suppose that this is not the case and choose a maximal counterexample $\mathbf{e}_{\max }$ with respect to $\prec$. When expanding $\mathbb{L} \mathbb{L}_{\mathbf{e}_{\text {max }}, \mathbf{e}_{0}, y}$ in terms of $\left\{S\left(\underline{w}^{\mathbf{f}}, \underline{w}^{\mathbf{f}_{1}}\right)\right\}$ we then have that the coefficient of $S\left(\underline{w}^{\mathbf{e}_{\text {max }}}, \underline{w}^{\mathbf{e}_{0}}\right)$ is nonzero, by the remarks after (5.6). On the other hand, when expanding any $\mathbb{L}_{\mathbf{e}_{1}, \mathbf{e}_{0}, y}$ in terms of $\left\{S\left(\underline{w}^{\mathbf{f}}, \underline{w}^{\mathbf{f}_{1}}\right)\right\}$ only terms with $\mathbf{f} \preceq \mathbf{e}_{1}$ and $\mathbf{f}_{1} \preceq \mathbf{e}_{0}$ appear with nonzero coefficient, as follows once again by the remarks after (5.6). Hence, these $\mathbb{L L}_{\mathbf{e}_{1}, \mathbf{e}_{0}, y}$ do not contribute to the coefficient of $S\left(\underline{w}^{\mathbf{e}_{\text {max }}}, \underline{w}^{\mathbf{e}_{0}}\right)$. Finally, for the basis elements $\mathbb{L L}_{\mathbf{e}_{2}, \mathbf{e}_{3}, z}$ appearing in the lower terms of (5.11) we have that $z<y$ and so these elements also do not contribute to the coefficient of $S\left(\underline{w}^{\mathbf{e}_{\max }}, \underline{w}^{\mathbf{e}_{0}}\right)$. All in all we get that the coefficient of $S\left(\underline{w}^{\mathbf{e} \text { max }}, \underline{w}^{\mathbf{e}_{0}}\right)$ on the right hand side of (5.11) is nonzero, which is the desired contradiction, by (5.10). Thus indeed we have proved that

$$
L_{i} \mathbb{L} \mathbb{L}_{\mathbf{e}_{\mathbf{e}, \mathbf{e}_{0}, y}}=\sum_{\substack{\mathbf{e}_{1} \in \operatorname{Tab}(y) \\ \mathbf{e}_{1} \preceq \mathbf{e}}} a_{\mathbf{e}_{1}} \mathbb{L} \mathbb{L}_{\mathbf{e}_{1}, \mathbf{e}_{0}, y}+\text { lower terms. }
$$

In order to determine the coefficient $a_{\mathbf{e}}$ of this expansion, we consider the coefficient of $S\left(\underline{w}^{\mathbf{e}}, \underline{w}^{\mathbf{e}}\right)$ on both sides of (5.12) and use (5.8). We find that $a_{\mathbf{e}}=c_{\mathbf{e}}(i)$ and so the Theorem is proved.

Let us illustrate the Theorem with the example $\underline{w}:=s s s$, with $y=s$ and $\mathbf{e}=(1,0,0)$. The corresponding light leaves diagram

$$
\text { h) }
$$

is the last one of (4.8), denoted $E 4$ in (5.4) and therefore the maximal diagram appearing in (4.8) with respect to path dominance. Now, applying $L_{1}$ to it gives the diagram denoted $E 3$, that is

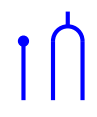

which is in accordance with the Theorem since we have $c_{\mathbf{e}}(1)=0$ for the content function.

Let us also calculate the action of $L_{2}$ on (5.13). Let $\delta \in \mathfrak{h}^{*}$ be the element coming from Demazure surjectivity. Then, using the polynomial and one-colour relations (3.3), (3.4), (3.6) we get that the 
action of $L_{2}$ on $(\underline{5.13)}$ is

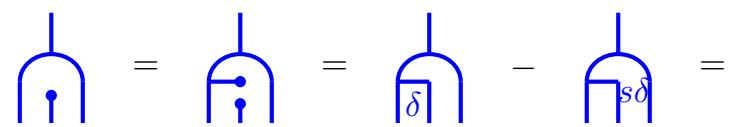

$$
\begin{aligned}
& s \delta-\delta !-\uparrow \bigcap \mid
\end{aligned}
$$

which is in accordance with the Theorem since $s \delta-\delta=-\alpha=s \alpha$.

Returning to the general situation of Definition 5.1, we define

$$
\operatorname{Tab}(\Lambda):=\bigcup_{\lambda \in \Lambda} \operatorname{Tab}(\lambda)
$$

We then extend the partial orders $\leq$ on the $\operatorname{Tab}(\lambda)$ 's to a partial order on Tab $(\Lambda)$ by the rule: $s<t$ if either $s, t \in \operatorname{Tab}(\lambda)$ and $s<t$ of if $s \in \operatorname{Tab}(\lambda), t \in \operatorname{Tab}(\mu)$ and $\lambda<\mu$. With this notation, Mathas formulated in 21 the following separation condition:

Definition 5.3. Let $\mathbf{L}=\left\{L_{1}, L_{2}, \ldots, L_{k}\right\}$ be a family of JM-elements as in Definition 5.1 . Then $\mathbf{L}$ is said to separate $\operatorname{Tab}(\Lambda)$ if for all $s, t \in \operatorname{Tab}(\Lambda)$ satisfying $s<t$, there exist $i$ such that $c_{s}(i) \neq c_{t}(i)$.

An important consequence of the separation condition in the following Proposition, see [21].

Proposition 5.4. Suppose that $\mathbf{L}$ is a family of JM-elements for a cellular algebra A. If $\mathbf{L}$ satisfies the separation condition, then $A$ is semisimple.

Our next result is an application of this Proposition. This result is also implicit in [5].

Proposition 5.5. The family of JM-elements $\mathbf{L}$ from Theorem 5.2 satisfies the separation condition over $Q$. In particular $A_{\underline{w}}$ is semisimple over $Q$.

Proof: $\quad$ Let $\mathbf{e}=\left(e_{1}, \ldots, e_{k}\right) \in \operatorname{Tab}(y)$ and $\mathbf{f}=\left(f_{1}, \ldots, f_{k}\right) \in \operatorname{Tab}\left(y_{1}\right)$. Let $i$ be minimal such that $e_{i} \neq f_{i}$. Then the symbols for $\mathbf{e}$ and $\mathbf{f}$ at position $i$ is either $U$ in both cases or $D$ in both cases. Thus, either one of them is $U 0$ and the other $U 1$ or one of them is $D 0$ and the other $D 1$. It now follows from (5.5) that the contents are different.

Remark. Note that the proof of the Proposition actually shows a separation condition which is stronger than the one given in Definition 5.1. Namely, for any distinct $\mathbf{e}, \mathbf{f} \in \operatorname{Tab}(\Lambda)$ there is an $i$ such that $c_{\mathbf{e}}(i) \neq c_{\mathbf{f}}(i)$.

\section{Determinant formula}

Let $A$ be a cellular algebra over $\mathbb{k}$ with cell datum $(\Lambda$, Tab, $C$ ). By the general theory developed in [8] there is a canonical family of $A$-modules $\{\Delta(\lambda) \mid \lambda \in \Lambda\}$, called the cell modules for $A$. Each cell module $\Delta(\lambda)$ is equipped with a natural symmetric, bilinear, $A$-invariant form $\langle\cdot, \cdot\rangle_{\lambda}$, given in terms of the cell datum. If $\mathbb{k}$ is a field, the form can be used to classify the irreducible modules for $A$. To be precise, the irreducible $A$-modules are in correspondence with the subset of $\Lambda$ given by

$$
\Lambda_{0}:=\left\{\lambda \in \Lambda \mid\langle\cdot, \cdot\rangle_{\lambda} \neq 0\right\}
$$

where for $\lambda \in \Lambda_{0}$ the corresponding irreducible module is given by $L(\lambda):=\Delta(\lambda) / \operatorname{rad}\langle\cdot, \cdot\rangle_{\lambda}$. Here $\operatorname{rad}\langle\cdot, \cdot\rangle_{\lambda}$ is the radical in the usual sense of a bilinear form; it is an $A$-submodule of $\Delta(\lambda)$ because of the $A$-invariance of $\langle\cdot, \cdot\rangle_{\lambda}$.

In the case we are interested in, that is the $R$-algebra $A_{\underline{w}}$ endowed with the cell datum explained in the previous sections, the cell module $\Delta_{\underline{w}}(y)$ has basis $\left\{\mathbb{L}_{\underline{\underline{w}}, \mathbf{e}} \mid \mathbf{e} \in \operatorname{Tab}(y)\right\}$. Let $y \in \operatorname{rexp}_{s}$ be a fixed reduced expression for $\underline{w}^{\mathbf{e}}$, the target object of the elements of $\left\{\mathbb{L}_{\underline{w}, \mathbf{e}} \mid \mathbf{e} \in \operatorname{Tab}(\bar{y})\right\}$. Then the value of the form $\left\langle\mathbb{L}_{\underline{w}, \mathbf{e}}, \mathbb{L}_{\mathbb{L}_{\underline{w}}, \mathbf{e}_{\mathbf{1}}}\right\rangle_{y}$ is by definition the coefficient of $\mathbb{L}_{\underline{y},(\mathbf{1}, \ldots, \mathbf{1}),(\mathbf{1}, \ldots, \mathbf{1})}$ when $\mathbb{L}_{\underline{w}, \mathbf{e}} \mathbb{L}_{\underline{w}, \mathbf{e}_{\mathbf{1}}}^{*}$ 
is expanded in the light leaves basis. Recall that $\mathbb{L}_{\underline{y}},(\mathbf{1}, \ldots, \mathbf{1}),(\mathbf{1}, \ldots, \mathbf{1})$ is not uniquely defined, but since $y \in \operatorname{rexp}_{s}$ a possible simple choice is the identity morphism. Note that the radical of the form is independent of the particular choice of $\mathbb{L}_{\underline{y},(\mathbf{1}, \ldots, \mathbf{1}),(\mathbf{1}, \ldots, \mathbf{1})}$.

Let us illustrate the form on a couple of examples. We consider the one-colour case $A_{\underline{s s}}$ where the basis of the cell module $\Delta_{\underline{s s}}(1)$ is

$$
\uparrow \bigcap
$$

We then get the following values of the bilinear form by taking the coefficient of the empty diagram

$$
\langle\boldsymbol{\imath}, \boldsymbol{\uparrow} \boldsymbol{\eta}\rangle_{1}=\alpha^{2} \quad\langle\bigcap, \bigcap\rangle_{1}=0 \quad\langle\bigcap, \boldsymbol{\uparrow}\rangle_{1}=\alpha
$$

Thus the determinant $\operatorname{det}\langle\cdot, \cdot\rangle_{1}$ of $\langle\cdot, \cdot\rangle_{1}$ is $-\alpha^{2}$. In particular, since $-\alpha^{2}$ is a unit in $Q$ we get that $L_{\underline{s s}}(1)=\Delta_{\underline{s s}}(1)$ which we of course already knew from the semisimplicity results of the previous section. On the other hand, if we were working over a ground field $\mathbb{k}$ in which $\alpha$ is specialized to 0 then $\langle\cdot, \cdot\rangle_{1}=0$ and we would have $1 \notin \Lambda_{0}$.

We next consider the cell module $\Delta_{\underline{s s}}(s)$ with diagram basis

$$
\text { १门. }
$$

We then get the following values of the bilinear form

$$
\langle\uparrow \mid \boldsymbol{\imath}\rangle_{s_{1}}=\alpha \quad\langle\bigcap, \hat{\bigcap}\rangle_{s_{1}}=0 \quad\langle\bigcap, \mathfrak{l}\rangle_{s_{1}}=1
$$

Thus the determinant $\operatorname{det}\langle\cdot, \cdot\rangle_{s}$ of $\langle\cdot, \cdot\rangle_{s}$ is 1 and $\Delta_{\underline{s s}}(s)$ is irreducible, even in specializations.

The billinear forms we are here considering coincide with the intersection forms from [15] and [34, see also [4] in the bimodule setting. In these references, the forms are further decomposed using the grading. Our diagonalization methods, to be explained next, only work in the ungraded case.

In complete generality the form $\langle\cdot, \cdot\rangle_{\lambda}$ is very difficult to control, but for a cellular algebra with a separating family $\mathbf{L}$ of JM-elements, there is at least a diagonalization strategy which can be used to calculate the determinant $\operatorname{det}\langle\cdot, \cdot\rangle_{\lambda}$ of $\langle\cdot, \cdot\rangle_{\lambda}$. Let us briefly explain this strategy, using section 3 of 21] as a reference. Let $A$ be the cellular algebra in question, defined over a field $\mathbb{k}$, and admitting a separating family $\mathbf{L}=\left\{L_{1}, L_{2}, \ldots, L_{k}\right\}$ of JM-elements. Let the cell modules be $\Delta(\lambda)$ for $\lambda \in \Lambda$. For $i=1, \ldots, k$ set $\mathcal{C}(i):=\left\{c_{t}(i) \mid t \in \operatorname{Tab}(\Lambda)\right\}$ where $\operatorname{Tab}(\Lambda)$ is as in (5.16). Suppose that $s \in \operatorname{Tab}(\lambda)$ and set

$$
F_{s}:=\prod_{i=1, \ldots, k} \prod_{\substack{c \in \mathcal{C}(i) \\ c \neq c_{s}(i)}} \frac{L_{i}-c}{c_{s}(i)-c} \in A
$$

see Definition 3.1 of [21]. Then we have that $F_{s} \neq 0$ and

$$
L_{i} F_{s}=c_{s}(i) F_{s}, \quad \sum_{s \in \operatorname{Tab}(\Lambda)} F_{s}=1 \quad \text { and } \quad F_{s} F_{t}=\delta_{s t} F_{s}
$$

where $\delta_{s t}$ is the Kronecker delta. Define the seminormal basis elements as $f_{s t}:=F_{s} C_{s t}^{\lambda} F_{t} \in A$ for $s, t \in \operatorname{Tab}(\lambda)$. For each $\lambda \in \Lambda$ fix some $t_{0} \in \operatorname{Tab}(\lambda)$ and define $C_{s}^{\lambda}=C_{s t_{0}}^{\lambda}$ and $f_{s}^{\lambda}=f_{s t_{0}}$. Then $\left\{f_{s}^{\lambda} \mid s \in \operatorname{Tab}(\lambda)\right\}$ is the seminormal basis for $\Delta(\lambda)$. Defining $\gamma_{s} \in \mathbb{k}$ by

$$
\gamma_{s}:=\left\langle f_{s}^{\lambda}, C_{s}^{\lambda}\right\rangle_{\lambda}
$$

we arrive at the following orthogonality statement

$$
\left\langle f_{s}^{\lambda}, f_{t}^{\lambda}\right\rangle_{\lambda}= \begin{cases}\gamma_{s} & \text { if } s=t \\ 0 & \text { otherwise }\end{cases}
$$

and determinant formula

$$
\operatorname{det}\langle\cdot, \cdot\rangle_{\lambda}=\prod_{s \in \operatorname{Tab}(\lambda)} \gamma_{s}
$$


Let us now apply this theory to our cellular algebra $A_{\underline{w}}$, defined in terms of $\underline{w}=s_{i_{1}} s_{i_{2}} \cdots s_{i_{k}} \in \exp _{s}$. As we have already seen, $A_{\underline{w}}$ is a cellular algebra over $\underline{R}$. Let $A_{\underline{w}, Q}:=A_{\underline{w}} \otimes_{R} Q$. In this particular case there is a direct diagrammatic way of constructing the eigenspace idempotents $F_{\mathbf{e}} \in A_{\underline{w}, Q}$ which can be easily explained using the previous sections and already appears implicitly in [5]. Indeed, each $\mathbf{e} \in \operatorname{Tab}(\Lambda)=\{0,1\}^{k}$ gives rise to the following diagram $F_{\mathbf{e}}^{\text {diag }}$ in $\operatorname{Kar}\left(\mathcal{D}_{Q}\right)$

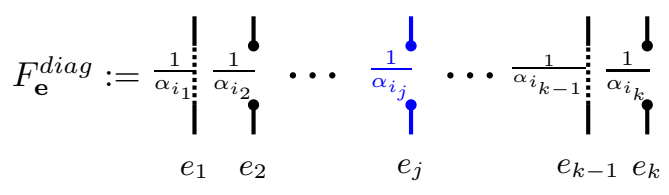

where $e_{j}=1$ corresponds to ${ }^{\frac{1}{\alpha_{i_{j}}}}$ and $e_{j}=0$ corresponds to ${ }^{\frac{1}{\alpha_{i_{j}}}}$ at the $j$ 'th position, and the colours of the lines are adjusted to $\underline{w}$. For example, in the above picture $e_{1}=1, e_{2}=0$ and $s_{j}$ is blue. Let us show the equality $F_{\mathrm{e}}^{\text {diag }}=\bar{F}_{\mathrm{e}}$ :

Lemma 6.1. For all $\mathbf{e} \in \operatorname{Tab}(\Lambda)$ we have that

$$
F_{\mathrm{e}}^{\text {diag }}=F_{\mathbf{e}}
$$

Proof: We first check that $F_{\mathbf{e}}^{\text {diag }} \neq 0$. Multiplying $F_{\mathbf{e}}^{\text {diag }}$ at the $j$ 'th position on top and bottom with $\vdots$ if $e_{j}=1$, and on top with $\boldsymbol{\varphi}$ and on bottom with $\boldsymbol{d}$ if $e_{j}=0$, and reducing the result via relations (3.3), (3.12) and (3.20), we arrive at $\lambda \cdot 1_{\underline{w}}$ where $\lambda \in Q^{\times}$and where $1_{\underline{w}}$ is the identity element of $\operatorname{End}_{\mathcal{D}^{s t d}}\left(\underline{w}^{\mathbf{e}}\right)$. But by (3.16) and the comments following (3.16), we have that $\operatorname{End}_{\mathcal{D}^{s t d}}\left(\underline{w}^{\mathbf{e}}\right) \neq 0$, and so also $1_{\underline{w}^{\mathrm{e}}} \neq 0$. Hence $F_{\mathrm{e}}^{\text {diag }} \neq 0$, as claimed.

Let $\mathcal{L}_{Q}$ be the subalgebra of $A_{\underline{w}, Q}$ generated by $\left\{L_{1}, L_{2} \ldots, L_{k}\right\}$. Then it follows from the relations (3.19) and (3.12) that $F_{\mathbf{e}}^{\text {diag }} \in \mathcal{L}_{Q}$. On the other hand, from (6.7) we have that $L_{i}=\sum_{\mathbf{e} \in \operatorname{Tab}(\Lambda)} c_{\mathbf{e}}(i) F_{\mathbf{e}}$ and so $\mathcal{L}_{Q}$ can also be described as the subalgebra of $A_{\underline{w}, Q}$ generated by the $F_{\mathbf{e}}$ 's. From this we obtain an expansion

$$
F_{\mathbf{e}}^{d i a g}=\sum_{\mathbf{f} \in \operatorname{Tab}(\Lambda)} \lambda_{\mathbf{f}} F_{\mathbf{f}}, \lambda_{\mathbf{f}} \in Q .
$$

We next observe that the formulas in (6.7) also hold when $F_{\mathbf{e}}$ is replaced by $F_{\mathbf{e}}^{\text {diag }}$. Indeed, the first formula in (6.7), involving the content function, is a consequence of the relations (3.3), (3.12) and (3.18), whereas the two other formulas follow from the relations (3.18) and (3.19).

Now by Proposition [5.5] and the Remark following it, there is for each $\mathbf{f} \neq \mathbf{e}$ an $i_{\mathbf{f}} \in\{1,2, \ldots, k\}$ such that $c_{\mathbf{e}}\left(i_{\mathbf{f}}\right) \neq c_{\mathbf{f}}\left(i_{\mathbf{f}}\right)$. Hence multiplying each side of (6.13) by $\prod_{\mathbf{f} \neq \mathbf{e}}\left(L_{i}-c_{\mathbf{f}}\left(i_{\mathbf{f}}\right)\right)$ we get

$$
\prod_{\mathbf{f} \neq \mathbf{e}}\left(c_{\mathbf{e}}\left(i_{\mathbf{f}}\right)-c_{\mathbf{f}}\left(i_{\mathbf{f}}\right)\right) F_{\mathbf{e}}^{\text {diag }}=\prod_{\mathbf{f} \neq \mathbf{e}}\left(c_{\mathbf{e}}\left(i_{\mathbf{f}}\right)-c_{\mathbf{f}}\left(i_{\mathbf{f}}\right)\right) \lambda_{\mathbf{e}} F_{\mathbf{e}} \Longleftrightarrow F_{\mathbf{e}}^{\text {diag }}=\lambda_{\mathbf{e}} F_{\mathbf{e}}
$$

But both $F_{\mathbf{e}}^{\text {diag }}$ and $F_{\mathbf{e}}$ are nonzero idempotents and so $\lambda_{\mathbf{e}}=1$, which shows (6.12).

We may now use $F_{\mathbf{e}}^{\text {diag }}$ to calculate $\gamma_{\mathbf{e}} \in Q$. Recall that we have fixed $\underline{w}=s_{j_{1}} s_{j_{2}} \cdots s_{j_{k}}$ and $\mathbf{e} \in \operatorname{Tab}(y)$. Let $i \in\{1, \ldots, k\}$ and define $w^{<i}:=s_{j_{1}}^{e_{1}} s_{j_{2}}^{e_{2}} \ldots s_{j_{i-1}}^{e_{i-1}}$. Let $M$ be the symbol of $\mathbf{e}$ at the $i$ 'th position and define for $i \in\{1, \ldots, k\}$ the element $\epsilon_{\mathbf{e}}^{i} \in Q$ by the formula

$$
\epsilon_{\mathbf{e}}^{i}= \begin{cases}w^{<i} \alpha_{j_{i}} & \text { if } T=U 0 \\ \left(w^{<i} \alpha_{j_{i}}\right)^{-1} & \text { if } T=D 0 \\ 1 & \text { if } T=U 1 \\ -1 & \text { if } T=D 1\end{cases}
$$

We now claim that $\epsilon_{\mathbf{e}}^{i}=\gamma_{\mathbf{e}}^{i}$. Assuming this claim is true we would obtain the promised determinant formula. It expresses the determinant $\operatorname{det}\langle\cdot, \cdot\rangle_{y}$ in terms of a purely Coxeter group combinatorial calculation, and in particular avoids any diagram calculations. Here is the precise formula and the proof of the claim: 
Proposition 6.2. In the above notation we have that $\epsilon_{\mathbf{e}}=\gamma_{\mathbf{e}}$ and $\gamma_{\mathbf{e}}=\prod_{i} \epsilon_{\mathbf{e}}^{i}$ and so

$$
\operatorname{det}\langle\cdot, \cdot\rangle_{y}=\prod_{\mathbf{e} \in \operatorname{Tab}(y), i \in\{1, \ldots, k\}} \epsilon_{\mathbf{e}}^{i}
$$

Proof: Let us warm up by considering the $A_{2}$-example in which $\mathbf{e}=(1,0,0), S:=\{\alpha, \beta\}, m_{\alpha \beta}=3$ and $\underline{w}:=s_{\alpha} s_{\beta} s_{\alpha}$. The corresponding light leaves diagram is given by $(U 1, U 0, D 0)$, it is

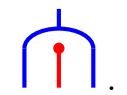

By the definitions, in order to calculate $\gamma_{\mathbf{e}}$ we should expand the following diagram in terms of the light leaves basis and take the coefficient of the identity diagram

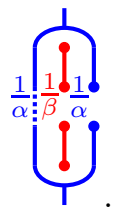

But applying the relations for $\operatorname{Kar}\left(\mathcal{D}_{Q}\right)$ we get that

$$
\underbrace{1}_{1}=\frac{1}{\alpha}=\beta \frac{1}{\alpha}-\frac{1}{\alpha} \beta \frac{1}{\alpha}
$$

where we used (3.19) for the second equality. Applying the polynomial relation (3.4) once to this, we find that $\gamma_{\mathbf{e}}$ is equal to $s\left(\beta \frac{1}{\alpha}\right)$, as claimed.

Let us now turn to the general case. Let us consider an $\mathbf{e} \in \operatorname{Tab}(y)$ and let $e_{i}$ be the $i$ 'th coordinate of $\mathbf{e}$. If the corresponding symbol is $M=U 0$, then the situation is exactly as for the $e_{2}$-term of (6.19), and the contribution to $\gamma_{\mathbf{e}}$ is $w^{<i} \alpha_{i}$ as claimed. If the corresponding symbol is $M=D 0$, then the situation is exactly as for the $e_{3}$-term of (6.19), adding a trivalent vertex, and the contribution to $\gamma_{\mathbf{e}}$ is $\left(w^{<i} \alpha_{i}\right)^{-1}$ as claimed.

If $T=U 1$ then there are two possibilities. Either the situation is as for $e_{1}$ in (6.19) where the dashed line extends to the top and bottom of the diagram and so the contribution to $\gamma_{\mathbf{e}}$ will be 1 , as claimed. Or alternatively, the dashed line turns around and eventually runs into a later $D 1$. This situation is not represented in (6.19) but appears in the example $\underline{w}:=s s, \mathbf{e}=(1,1)$ with symbols $(U 1, D 1)$. The light leaves morphism is in this case $\bigcap$ and so we should take the coefficient of the empty diagram in

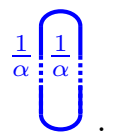

By (3.12), (3.13), (3.21) and (3.22) the value of this is -1 , which is therefore the combined contribution of the $U 1$ and $D 1$ symbols in this case, as claimed. The general case is done the same way.

Remark. Note that $\gamma_{\mathbf{e}}^{i} \in Q$, but even so $\operatorname{det}\langle\cdot, \cdot\rangle_{y} \in R$, of course.

Example. Let us take $\underline{w}:=$ sss. In (4.7) we have given the basis for $\Delta(1)$ and in (4.8) the basis for $\Delta(s)$. But using the symbols given in (4.6) we get easily via the Proposition, without drawing diagrams, that $\operatorname{det}\langle\cdot, \cdot\rangle_{1}=\alpha^{4}$ and $\operatorname{det}\langle\cdot, \cdot\rangle_{s}=1$.

Remark. It follows from the Proposition that $\operatorname{det}\langle\cdot, \cdot\rangle_{y}$ is always a product of roots.

Remark. Note that by general principles the zero'th homogeneous part $A_{\underline{w}}^{0}$ of $A_{\underline{w}}$ is also a cellular algebra. Unfortunately, as already mentioned in the introduction, our formula is not compatible with the grading on $A_{\underline{w}}$, and in particular is does not give rise to a determinant formula for the cell modules for $A_{\underline{w}}^{0}$. The reason is that the JM-elements $\left\{L_{i}\right\}$ are homogeneous of degree 2 and hence cannot be used to diagonalize the cell modules for $A_{\underline{w}}^{0}$. 


\section{$7 \quad$ A Shapovalov-like expression for $\operatorname{det}\langle\cdot, \cdot\rangle_{y}$}

The usefulness of determinant expressions in representation theory depends on the property that they can be rewritten in terms of characters or dimensions of other cell modules. This rewriting was first achieved by Shapovalov and Jantzen for Verma modules for complex semisimple Lie algebras, see [30] and 12. Later, similar rewritings were found for Weyl modules for algebraic groups by Jantzen and Andersen, for Specht modules for the symmetric groups by Schaper, James, Murphy and so on, see for example [1], 2], 11. This section is devoted to a rewriting of $\operatorname{det}\langle\cdot, \cdot\rangle_{y}$ in this spirit. A major difference between our case and the above mentioned cases is that we do not have a closed formula for the dimension of the cell module.

Our argument will be an induction on the length of $\underline{w}$ using Proposition 6.2. In this sense, it is closest to the argument given by James and Murphy for the symmetric group, see [11. Just like in [11] we also need a branching rule for $\Delta_{\underline{w}}(y)$. For $A_{\underline{w}}$ this branching rule was discovered by D. Plaza in [26] in the bimodule setting, but, as we shall shortly see, it carries over to the diagrammatical setting.

Recall that we have fixed the expression $\underline{w}=s_{j_{1}} \cdots s_{j_{k}}$. Set $\alpha:=\alpha_{j_{k}}$ and $s:=s_{j_{k}}$, where $j_{k}$ corresponds to the color blue, say. For any $x \in W$ we define $x^{\prime}:=x s$. There is a straightforward embedding $A_{\underline{w^{\prime}}} \subseteq A_{\underline{w}}$ defined diagrammatically by adding a through blue line to the right of a diagram in $A_{\underline{w^{\prime}}}$, as illustrated below

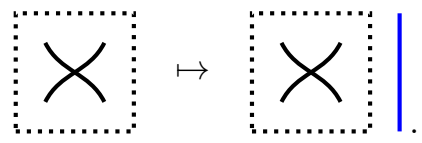

Letting $A_{\underline{w^{\prime}}}$-mod and $A_{\underline{w}}$-mod denote the module categories for $A_{\underline{w^{\prime}}}$ and $A_{\underline{w}}$, we get an associated restriction functor

$$
A_{\underline{w^{-}}} \text {mod } \rightarrow A_{\underline{w^{\prime}}} \text { mod, } M \mapsto \operatorname{Res} M
$$

In general, we use from now on the convention that $\Delta_{\underline{z}}(y):=0$ if $y \not \leq z$. The following branching rule was found by D. Plaza in the bimodule setting, see [27. We now state and prove it in the diagrammatical setting.

Theorem 7.1. Assume that $y \leq w$.

1) If $y^{\prime}>y$ then there is a short exact sequence of $A_{\underline{w^{\prime}}}$-modules

$$
0 \rightarrow \Delta_{\underline{w}^{\prime}}(y) \rightarrow \operatorname{Res} \Delta_{\underline{w}}(y) \rightarrow \Delta_{\underline{w}^{\prime}}\left(y^{\prime}\right) \rightarrow 0 .
$$

2) If $y^{\prime}<y$ then there is a short exact sequence of $A_{\underline{w^{\prime}}}$-modules

$$
0 \rightarrow \Delta_{\underline{w}^{\prime}}\left(y^{\prime}\right) \rightarrow \operatorname{Res} \Delta_{\underline{w}}(y) \rightarrow \Delta_{\underline{w}^{\prime}}(y) \rightarrow 0 .
$$

Remark. In [26 the sequences are considered as sequences of graded modules over the graded algebra $A_{\underline{w}^{\prime}}$. In view of the last remark of the previous section we here ignore the grading.

Proof: Let first $M$ be the symbol at the $k^{\prime}$ th position of any $\mathbf{e} \in \operatorname{Tab}(y)$. By definition, if $y^{\prime}>y$ then $M$ is either $U 0$ or $D 1$ whereas if $y^{\prime}<y$ then $M$ is either $U 1$ or $D 0$.

Let us first consider the case $y^{\prime}>y$. The light leaves diagrams $\mathbb{L L}_{\underline{w}, \mathbf{e}}$ satisfying $M=U 0$ are exactly those with $\mathfrak{l}$ on the right. They define an $A_{\underline{w^{\prime}}}$-module isomorphic to $\Delta_{\underline{w}^{\prime}}(y)$ where the isomorphism is given by adding $I_{\text {to }}$ the right. On the other hand, the light leaves diagrams $\mathbb{L}_{\underline{w}, \mathbf{e}}$ satisfying $M=D 1$ are in bijection with the light leaves basis for $\Delta_{\underline{w^{\prime}}}\left(y^{\prime}\right)$ where the bijection can be described by bending up the last line, thus transforming a $\cap$ into a vertical blue line $\mathrm{l}$. For example, in the $A 1$-case given in (4.7) the first two diagrams end in $M=U 0$ and the last two diagrams end in $M=D 1$ and these become, after bending up, the diagrams of (6.4).

The bending up also works well at the module level. Indeed, let us extend it to a linear map $\pi: \Delta_{\underline{w}}(y) \rightarrow \Delta_{\underline{w}^{\prime}}\left(y^{\prime}\right)$ given diagrammatically via

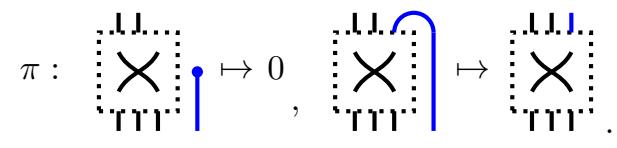


Then $\operatorname{ker} \pi=\Delta_{\underline{w^{\prime}}}(y), \operatorname{im} \pi=\Delta_{\underline{w}^{\prime}}(y)$. Moreover, $\pi$ is an $A_{\underline{w^{\prime}}}$-linear map as follows from the diagrammatical identity

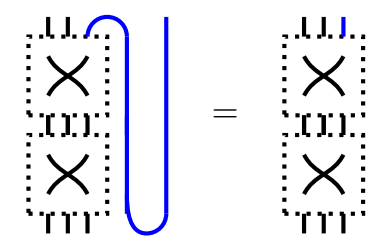

where the left hand side represents $\pi(a v)$ for $a \in A_{\underline{w}^{\prime}}$ and $v \in \Delta_{\underline{w}}(y)$ and the right hand side $a \pi(v)$. Upon expanding the left hand side in terms of the light leaves basis for $\Delta_{\underline{w}}(y)$ the terms with a blue dot in the upper right corner are mapped to zero under $\pi$ and are also viewed as zero in the right hand side.

In the case $y^{\prime}<y$, the e's with $M=U 1$ correspond to diagrams with a blue line $I$ to the right. These are in correspondence with a basis for the $A_{\underline{w^{\prime}}}$-module $\Delta_{\underline{w^{\prime}}}\left(y^{\prime}\right)$, where the correspondence is given by deleting the blue line. The remaining diagrams, corresponding to $M=D 0$, have a trivalent vertex on the right. These diagrams are in bijection with the light leaves basis for $\Delta_{\underline{w}^{\prime}}(y)$, where the bijection is given by transforming the trivalent vertex to a line.

Also in this case, one checks that the maps work well at the module level.

In order to give the Shapovalov-like reformulation of the determinant, we shall assume that $\mathfrak{h}$ is of one of the following two types. For the first type $\mathfrak{h}$ is chosen as the geometric representation of $(W, S)$, considered in 10. Recall that this $\mathfrak{h}$ is a realization of $(W, S)$, in the above sense, defined over $\mathbb{R}$ and faithful as a $W$-representation. A root $\alpha \in \mathfrak{h}^{*}$ is by definition an element of the form $\alpha=w \alpha_{s}$ for $s \in S$ and $w \in W$. It is said to be positive, written $\alpha>0$, if $\alpha=\sum_{s \in S} \lambda_{s} \alpha_{s}$ for $\lambda_{s} \geq 0$ for all $s$. For $\alpha$ a root there is a well-defined associated reflection $s_{\alpha}$ defined by $s_{\alpha}=z s z^{-1}$ where $z \in W$ is chosen such that $\alpha=z \alpha_{s}$.

For the second type we shall assume that $(W, S)$ is the Weyl group of the root system $\Phi$ with Cartan matrix $A=\left(a_{s t}\right)_{s, t \in S}$. We let $\mathfrak{h}_{\mathbb{Z}}:=\oplus_{s \in S} \mathbb{Z} \alpha_{s}^{\vee}$ be the coroot lattice. Then $\mathfrak{h}_{\mathbb{Z}}$, together with $\left.\left\{\alpha_{s}^{\vee} \mid s \in S\right\} \subset \mathfrak{h}_{\mathbb{Z}}\right\}$ and $\left.\left\{\alpha_{s} \mid s \in S\right\} \subset \mathfrak{h}_{\mathbb{Z}}^{*}\right\}$, defines a realization of $(W, S)$ over $\mathbb{Z}$. Let $p>3$ be a prime. Then our second type of realization is $\mathfrak{h}:=\mathfrak{h}_{\mathbb{Z}} \otimes_{\mathbb{Z}} \overline{\mathbb{F}}_{p}$. This is the Cartan matrix representation of $W$. In $\mathfrak{h}_{\mathbb{Z}}$ we have the concept of roots and positive roots just as before.

Although other choices of realizations $\mathfrak{h}$ are possible, at present the above ones are sufficient for the applications we have in mind. We remark that both choices of realizations are Soergel realizations in the above sense. For the first $\mathfrak{h}$ this is a consequence of [18] and for the second $\mathfrak{h}$ it follows from the Appendix of [19; this is where $p>3$ is needed. Thus in both cases we obtain equivalences between the diagrammatic and bimodules categories, see [5].

Let us now return to the determinant $\operatorname{det}\langle\cdot, \cdot\rangle_{\lambda}$. We prove the following Theorem, giving the promised Shapovalov-like reformulation of the determinant.

Theorem 7.2. Let $\mathfrak{h}$ be as above. Then we have that

$$
\operatorname{det}\langle\cdot, \cdot\rangle_{y}= \pm \prod_{\beta>0, s_{\beta} y>y} \beta^{\operatorname{dim} \Delta_{\underline{w}}\left(s_{\beta} y\right)}
$$

Proof: The proof is a purely combinatorial transformation of the formula from Proposition 6.2 into the expression given in (7.7), using induction on the length $k$ of $\underline{w}$. The case $k=1$ is straightforward to check. Let us therefore assume that (7.7) holds for $k-1$ and verify it for $k$. We do it simultaneously for the two choices for $\mathfrak{h}$; for the second choice we work with $\mathfrak{h}_{\mathbb{Z}}$ and deduce the formula (7.7) for $\mathfrak{h}$

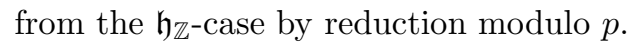

Suppose first that $y^{\prime}>y$. As mentioned in the proof of the previous Theorem 7.1, the e's ending in $U 0$ give rise to a basis for $\Delta_{\underline{w}}(y)$, whereas those ending in $D 1$ give rise to a basis for $\Delta_{\underline{w}}\left(y^{\prime}\right)$, by restriction to $\underline{w}^{\prime}$. Thus, by the inductive hypothesis and Proposition 6.2 we get that

$$
\operatorname{det}\langle\cdot, \cdot\rangle_{y}= \pm(y \alpha)^{\operatorname{dim} \Delta_{\underline{w^{\prime}}}(y)} \prod_{\beta>0, s_{\beta} y>y} \beta^{\operatorname{dim} \Delta_{\underline{w}^{\prime}}\left(s_{\beta} y\right)} \prod_{\beta>0, s_{\beta} y^{\prime}>y^{\prime}} \beta^{\operatorname{dim} \Delta_{\underline{w^{\prime}}}}\left(s_{\beta} y^{\prime}\right)
$$


Now using (7.3) and (7.4) we get that

$$
\operatorname{dim} \Delta_{\underline{w}}\left(s_{\beta} y\right)=\operatorname{dim} \Delta_{\underline{w}^{\prime}}\left(s_{\beta} y^{\prime}\right)+\operatorname{dim} \Delta_{\underline{w}^{\prime}}\left(s_{\beta} y\right)
$$

and so we would like to join the two last products of (7.8) into one product, running over $\beta>0$ with $s_{\beta} y>y$. As long as $\beta$ is in $R_{1}:=\left\{\beta>0 \mid s_{\beta} y>y\right.$ iff $\left.s_{\beta} y^{\prime}>y^{\prime}\right\}$ this is clearly possible. Let us therefore analyse the sets

$$
R_{2}:=\left\{\beta>0 \mid s_{\beta} y<y \text { and } s_{\beta} y^{\prime}>y^{\prime}\right\} \text { and } R_{3}:=\left\{\beta>0 \mid s_{\beta} y>y \text { and } s_{\beta} y^{\prime}<y^{\prime}\right\} .
$$

We first claim that $R_{2}=\emptyset$. Indeed, if $\beta \in R_{2}$, then $s_{\beta} y<y$ and so we get that $l\left(s_{\beta} y\right) \leq l(y)-1=$ $l\left(y^{\prime}\right)-2$, in contradiction with $s_{\beta} y^{\prime}>y^{\prime}$.

Let us then consider $\beta \in R_{3}$. Let $y=s_{i_{1}} \cdots s_{i_{m}}$ be a reduced expression for $y$. Then $y^{\prime}=s_{i_{1}} \cdots s_{i_{m}} s$ is a reduced expression for $y^{\prime}$. By the strong exchange condition, see Theorem 5.8 of [10], applied to $s_{\beta} y^{\prime}<y^{\prime}$, we get that either $s_{\beta} y^{\prime}=s_{i_{1}} \ldots \widehat{s_{i_{j}}} \cdots s_{i_{m}} s$ for some $j$, or $s_{\beta} y^{\prime}=s_{i_{1}} \cdots s_{i_{m}}$. The first case is impossible since it would imply that $s_{\beta} y=s_{i_{1}} \cdots \widehat{s_{i_{j}}} \cdots s_{i_{m}}<y$. The second case corresponds to $s_{\beta} y s=y$, or equivalently $s_{\beta}=y s y^{-1}$ and so $R_{3}=\{y \alpha\}$.

All in all, using (7.9) we find that the contribution to (7.8) from $R_{1}$ is

$$
\pm \prod_{\beta \in R_{1}, s_{\beta} y>y} \beta^{\operatorname{dim} \Delta_{\underline{w}^{\prime}}\left(s_{\beta} y\right)} \prod_{\beta \in R_{1}, s_{\beta} y^{\prime}>y^{\prime}} \beta^{\operatorname{dim} \Delta_{\underline{w^{\prime}}}\left(s_{\beta} y^{\prime}\right)}= \pm \prod_{\beta \in R_{1}} \beta^{\operatorname{dim} \Delta_{\underline{w}}\left(s_{\beta} y\right)} .
$$

The contribution from $R_{3}$ to (7.8) comes from the second factor. But since $s_{\beta} y=y^{\prime}$, its contribution together with the first factor of (17.8) is

$$
(y \alpha)^{\operatorname{dim} \Delta_{\underline{w^{\prime}}}(y)}(y \alpha)^{\operatorname{dim} \Delta_{\underline{w}^{\prime}}\left(y^{\prime}\right)}=(y \alpha)^{\operatorname{dim} \Delta_{\underline{w}}(y)} .
$$

Finally, since $R_{2}$ does not contribute and since $R_{1} \cup R_{3}=\left\{\beta>0 \mid s_{\beta} y>y\right\}$ we conclude that our formula (7.7) of the Theorem holds.

The case $y^{\prime}<y$ is treated the same way.

\section{Jantzen type filtrations and sum formulas.}

We construct in this section an analogue of the Jantzen filtration and, as an application of Theorem 7.2. obtain an associated sum formula. Recall that the original Jantzen filtration was constructed for Verma modules for semisimple complex Lie algebras. Although the combinatorial methods used here have little in common with those used in the representation theory of Lie algebras, ultimately the sum formulas rely in both cases on determinant expressions.

Let us first suppose that $\mathfrak{h}$ is the geometric representation of $(W, S)$, defined over the real numbers $\mathbb{R}$. Let $\mathcal{R}$ be the polynomial algebra $\mathcal{R}:=\mathbb{R}[x]$. Then $\mathcal{R}$ is a discrete valuation domain with maximal ideal $(x)$ and $x$-adic valuation which we denote by $\nu_{x}(\cdot)$. Recall that $R=\oplus_{m} S^{m}\left(\mathfrak{h}^{*}\right)$. It may be identified with the polynomial algebra over $\mathbb{R}$ in $|S|$ variables and hence there is a natural algebra homomorphism $\varphi: R \rightarrow \mathcal{R}$ satisfying $\nu_{x}(\varphi(\beta))=1$ for all roots $\beta$.

Let us consider the base change from $R$ to $\mathcal{R}$

$$
A_{\underline{w}, \mathcal{R}}:=A_{\underline{w}} \otimes_{R} \mathcal{R} .
$$

By the general cellular algebra theory, we then know that $A_{\underline{w}, \mathcal{R}}$ is also a cellular algebra, with cell modules $\Delta_{\underline{w}, \mathcal{R}}(y):=\Delta_{\underline{w}}(y) \otimes_{R} \mathcal{R}$. Let us denote the associated bilinear form on $\Delta_{\underline{w}, \mathcal{R}}(y)$ by $\langle\cdot, \cdot\rangle_{y}$ as well. Motivated by Jantzen's original work we now define

$$
\Delta_{\underline{w}, \mathcal{R}}^{i}(y):=\left\{v \in \Delta_{\underline{w}, \mathcal{R}}(y) \mid\langle v, w\rangle_{y} \in x^{i} \mathcal{R} \text { for all } w \in \Delta_{\underline{w}, \mathcal{R}}(y)\right\} .
$$

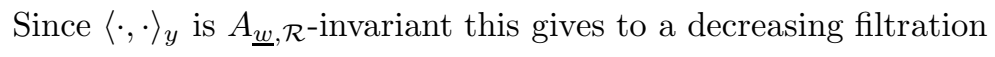

$$
\Delta_{\underline{w}, \mathcal{R}}(y)=\Delta_{\underline{w}, \mathcal{R}}^{0}(y) \supseteq \Delta_{\underline{w}, \mathcal{R}}^{1}(y) \supseteq \Delta_{\underline{w}, \mathcal{R}}^{2}(y) \supseteq \cdots
$$

of $A_{\underline{w}}, \mathcal{R}$-modules. 
From $\mathcal{R}$ we can further extend scalars to $\mathbb{R}$ via the homomorphism $\mathcal{R} \rightarrow \mathcal{R} /(x) \cong \mathbb{R}$. We denote the corresponding cellular algebra by $A_{\underline{w}, \mathbb{R}}$ and its cell modules by $\Delta_{\underline{w}, \mathbb{R}}(y):=\Delta_{\underline{w}}, \mathcal{R}(y) \otimes_{\mathcal{R}} \mathbb{R}$. We denote the bilinear form on $\Delta_{\underline{w}, \mathbb{R}}(y)$ by $\left.\overline{\langle\cdot}, \cdot\right\rangle_{y}$, too.

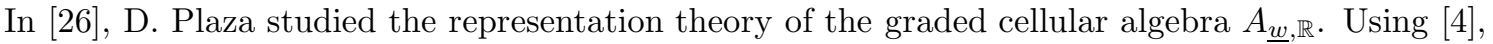
he showed that the nonzero graded decomposition numbers for $A_{\underline{w}, \mathbb{R}}$ are Kazhdan-Lusztig polynomials for $W$. In other words, $A_{\underline{w}, \mathbb{R}}$ is a highly interesting non-semisimple algebra. Although it is not quasi-hereditary, it still has many features in common with category $\mathcal{O}$ for a complex semisimple Lie algebra.

The inclusion $\Delta_{\underline{w}, \mathcal{R}}^{i}(y) \subseteq \Delta_{\underline{w}, \mathcal{R}}(y)$ induces a homomorphism $\Delta_{\underline{w}, \mathbb{R}}^{i}(y) \rightarrow \Delta_{\underline{w}, \mathbb{R}}(y)$ and we define $\Delta_{\underline{w}, \mathbb{R}}^{i}(y) \subseteq \Delta_{\underline{w}, \mathbb{R}}(y)$ as the image of this homomorphism. Via (‥3) this gives rise to a filtration of $A_{\underline{w}}, \mathbb{R}^{-m o d u l e s}$

$$
\Delta_{\underline{w}, \mathbb{R}}(y)=\Delta_{\underline{w}, \mathbb{R}}^{0}(y) \supseteq \Delta_{\underline{w}, \mathbb{R}}^{1}(y) \supseteq \Delta_{\underline{w}, \mathbb{R}}^{2}(y) \supseteq \cdots
$$

This filtration of $\Delta_{\underline{w}, \mathbb{R}}(y)$ is our analogue of the Jantzen filtration for Verma modules and the following Theorem is a weak analogue of Jantzen's sum formula, involving dimensions of the modules. It follows from the Theorem that the filtration is finite.

Theorem 8.1. We have that $\operatorname{dim} \Delta_{\underline{w}, \mathbb{R}}(y) / \Delta_{\underline{w}, \mathbb{R}}^{1}(y)=\operatorname{dim} \Delta_{\underline{w}, \mathbb{R}}(y) / \operatorname{rad}\langle\cdot, \cdot\rangle_{y}$. Moreover, the following sum formula holds

$$
\sum_{i>0} \operatorname{dim} \Delta_{\underline{w}, \mathbb{R}}^{i}(y)=\sum_{\beta>0, s_{\beta} y>y} \operatorname{dim} \Delta_{\underline{w}, \mathbb{R}}\left(s_{\beta} y\right) .
$$

Proof: The proof follows closely the proof in the classical case, see 'Key Lemma' of section 5.6 of 9 . For the reader's convenience we here sketch the argument. We first prove (8.5). Let $s:=\operatorname{rank} \Delta_{\underline{w}}, \mathcal{R}(y)$. Since $\mathcal{R}$ is a principal ideal domain, the matrix $M$ for $\langle\cdot, \cdot\rangle_{y}$ is equivalent to a diagonal $\mathcal{R}$-matrix $D:=\operatorname{diag}\left(d_{1}, \ldots, d_{s}\right)$, in other words there are invertible $\mathcal{R}$-matrices $A, B$ of sizes $s \times s$ such that $D=A M B$. For $j=1, \ldots, s$ we let $v_{j}$ be the basis vector corresponding to the $j$ 'th column of $D$ and we set $a_{j}:=\nu_{x}\left(d_{j}\right)$. Then $\Delta_{\underline{w}, \mathcal{R}}^{i}(y)$ is spanned by the elements $\left\{v_{j} \mid a_{j} \geq i\right\}$ together with the elements $\left\{x^{i-a_{j}} v_{j} \mid a_{j}<i\right\}$. The last elements vanish when tensoring over $\mathbb{R}$ and so the left hand side of (8.5) is $a_{1}+a_{2}+\ldots+a_{s}$. But this is equal to $\nu_{x}(\operatorname{det} D)$ which is equal to the right hand side of (8.5) by Theorem 7.2 and the assumption on $\varphi$.

The equality $\operatorname{dim} \Delta_{\underline{w}, \mathbb{R}}(y) / \Delta_{\underline{w}, \mathbb{R}}^{1}(y)=\operatorname{dim} \Delta_{\underline{w}, \mathbb{R}}(y) / \operatorname{rad}\langle\cdot, \cdot\rangle_{y}$ is proved similarly.

We next explain one of several ways to obtain a sum formula in the positive characteristic situation. Let $\mathfrak{h}$ be the Cartan matrix representation of a Weyl group $W$ over $\overline{\mathbb{F}}_{p}$, as introduced above. Choose this time $\mathcal{R}:=\mathbb{Z}_{(p)}$, the localization of $\mathbb{Z}$ at $p$. Then $\mathcal{R}$ is also a discrete valuation domain with valuation function that we denote $\nu_{p}(\cdot)$. Its maximal ideal is $p \mathcal{R}$ and we have $\mathcal{R} / p \mathcal{R} \cong \mathbb{F}_{p}$. Let $\varphi: \mathfrak{h}_{\mathbb{Z}} \rightarrow \mathbb{Z}$ be the homomorphism of $\mathbb{Z}$-modules given by $\varphi\left(\alpha_{s}\right):=p$ for the basis elements $\left\{\alpha_{s} \mid s \in S\right\}$ and denote also by $\varphi$ the homomorphism obtained from $\varphi$ by composing with the inclusion $\mathbb{Z} \subseteq \mathcal{R}$.

We define $A_{\underline{w}}, \mathcal{R}:=A_{\underline{w}} \otimes_{R} \mathcal{R}, A_{\underline{w}, \mathbb{F}_{p}}:=A_{\underline{w}, \mathcal{R}} \otimes_{\mathcal{R}} \mathbb{F}_{p}$ and so on, mimicking what we did before. We then obtain an $\bar{A}_{\underline{w}}, \mathcal{R}$-module filtration of $\Delta_{\underline{w}}, \mathcal{R}(y)$ via

$$
\Delta_{\underline{w}, \mathcal{R}}^{i}(y):=\left\{v \in \Delta_{\underline{w}}, \mathcal{R}(y) \mid\langle v, w\rangle_{y} \in p^{i} \mathcal{R} \text { for all } w \in \Delta_{\underline{w}, \mathcal{R}}(y)\right\}
$$

and this induces a filtration $\left\{\Delta_{\underline{w}, \mathbb{F}_{p}}^{i}(y) \mid i=0,1, \ldots\right\}$ of $\Delta_{\underline{w}, \mathbb{F}_{p}}(y)$ and hence also a filtration $\left\{\Delta_{\underline{w}, \overline{\mathbb{F}}_{p}}^{i}(y) \mid i=\right.$ $0,1, \ldots\}$ of $\Delta_{\underline{w}, \overline{\mathbb{F}}_{p}}(y)$ via $\Delta_{\underline{w}, \overline{\mathbb{F}}_{p}}^{i}(y):=\Delta_{\underline{w}, \mathbb{F}_{p}}^{i}(y) \otimes_{\mathbb{F}_{p}} \overline{\mathbb{F}}_{p}$. We get the following Theorem.

Theorem 8.2. We have that $\operatorname{dim} \Delta_{\underline{w}, \overline{\mathbb{F}}_{p}}(y) / \Delta_{\underline{w}, \overline{\mathbb{F}}_{p}}^{1}(y)=\operatorname{dim} \Delta_{\underline{w}, \overline{\mathbb{F}}_{p}}(y) / \operatorname{rad}\langle\cdot, \cdot\rangle_{y}$. Moreover, the following sum formula holds

$$
\sum_{i>0} \operatorname{dim} \Delta_{\underline{w}, \bar{F}_{p}}^{i}(y)=\sum_{\beta>0, s_{\beta} y>y} \nu_{p}(\varphi(\beta)) \operatorname{dim} \Delta_{\underline{w}, \bar{F}_{p}}\left(s_{\beta} y\right) .
$$

Proof: The proof is essentially the same as the proof of Theorem 8.1 .

In the classical theory the sum formula is formulated as an equality in the Grothendieck group and thus we would have expected (8.5) and (8.7) to hold at this level of generality. Our next goal is to 
show that in fact there is a variation of the above constructions for which (8.5) and (8.7) do hold at the Grothendieck group level.

As can be seen already in the one-colour calculations presented above, the algebra $A_{\underline{w}, \mathbb{R}}$ is not quasi-hereditary in general, in other words we have $\Lambda \neq \Lambda_{0}$ in general. Moreover, in general it appears to be difficult to determine $\Lambda_{0}$, and hence for the Grothendieck group to work well we need to change the setup.

As mentioned above, $\mathcal{D}$ is cellular category in the sense of Westbury. Let us recall his definition from 33:

Definition 8.3. Let $\mathbb{k}$ be a commutative ring with identity and let $\mathcal{C}$ be $a \mathbb{k}$-linear category with duality *. Then $\mathcal{C}$ is called a cellular category if there exists a poset $\Lambda$ and for each $\lambda \in \Lambda$ and each object $n$ in $\mathcal{D}$ a finite set $\operatorname{Tab}(n, \lambda)$ together with a map $\operatorname{Tab}(m, \lambda) \times \operatorname{Tab}(n, \lambda) \rightarrow H_{\mathcal{C}}(m, n),(S, T) \mapsto C_{S T}^{\lambda}$, satisfying $\left(C_{S T}^{\lambda}\right)^{*}=C_{T S}^{\lambda}$. These data satisfy that

$$
\left\{C_{S T}^{\lambda} \mid S \in \operatorname{Tab}(m, \lambda), T \in \operatorname{Tab}(n, \lambda), \lambda \in \Lambda\right\} \text { is a basis for } \operatorname{Hom}_{\mathcal{C}}(m, n)
$$

and for all $a \in H_{\mathcal{C}}(n, p), S \in \operatorname{Tab}(m, \lambda), T \in \operatorname{Tab}(n, \lambda)$

$$
a C_{S T}^{\lambda}=\sum_{S^{\prime} \in \operatorname{Tab}(p, \lambda)} r_{a}\left(S^{\prime}, S\right) C_{S^{\prime}, T}^{\lambda} \bmod A^{\lambda}
$$

where $A^{\lambda}$ is the span of $\left\{C_{S T}^{\mu} \mid \mu<\lambda, S \in \operatorname{Tab}(m, \mu), T \in \operatorname{Tab}(p, \mu)\right\}$.

As already mentioned in 33 , each object $m$ in a cellular category $\mathcal{C}$ gives rises to the cellular algebra $\operatorname{End}_{\mathcal{R}}(m)$. This fact can be generalized as follows. Let $I$ be any finite subset of the objects of $\mathcal{C}$ and define $\operatorname{End}_{\mathcal{C}}(I)$ as the $\mathbb{k}$-direct sum

$$
\operatorname{End}_{\mathcal{C}}(I):=\oplus_{m, n \in I} \operatorname{Hom}_{\mathcal{C}}(m, n) .
$$

Then $\operatorname{End}_{\mathcal{C}}(I)$ has a natural $\mathbb{k}$-algebra structure as follows

$$
g \cdot f:= \begin{cases}g f & \text { if } f \in \operatorname{Hom}_{\mathcal{C}}(m, n), g \in \operatorname{Hom}_{\mathcal{C}}(n, p) \text { for some } m, n, p \\ 0 & \text { otherwise. }\end{cases}
$$

Theorem 8.4. Let $\mathcal{C}$ be a cellular category as in Definition 8.3 and define for $\lambda \in \Lambda$ the set $\operatorname{Tab}(\lambda):=$ $\cup_{n \in I} \operatorname{Tab}(n, \lambda)$. Let for $S \in \operatorname{Tab}(\lambda), T \in \operatorname{Tab}(\lambda)$ the element $C_{S T}^{\lambda} \in \operatorname{End}_{\mathcal{C}}(I)$ be defined as the corresponding inclusion of $C_{S T}^{\lambda} \in \operatorname{Hom}_{\mathcal{C}}(m, n)$ in $\operatorname{End}_{\mathcal{C}}(I)$. Then these data define a cellular algebra structure on $\operatorname{End}_{\mathcal{C}}(I)$.

Proof: This is immediate from the definitions.

For our category $\mathcal{D}$ the objects are $\exp _{s}$ and the tab function is

$$
\operatorname{Tab}(\underline{w}, y):=\left\{\mathbf{e} \in\{0,1\}^{k} \mid \mathbf{e} \text { subexpression of } \underline{w} \text { expressing } y\right\} .
$$

Let us now choose an ideal $\pi$ in $W$, that is $x \in \pi, y<x \Rightarrow y \in \pi$, and let us also choose for all $y \in \pi$ an arbitrary $\underline{y} \in \operatorname{rexp}_{s}$ expressing $y$. We set $\underline{\pi}:=\{\underline{y} \mid y \in \pi\}$ and define the algebra

$$
A_{\pi}=A_{\underline{\pi}}:=\operatorname{End}_{\mathcal{D}}\left(\oplus_{y \in \pi} \underline{y}\right) .
$$

Then, according to the above Theorem we have that $A_{\pi}$ is a cellular algebra on the poset $\Lambda_{\pi}:=\pi$. For $y \in \Lambda_{\pi}$ the corresponding $\operatorname{Tab}(y)$ is $\operatorname{Tab}_{\pi}(y):=\bigcup_{z \in \pi} \operatorname{Tab}(\underline{z}, y)$. For $y \in \pi$ we denote by $\Delta_{\pi}(y)$ the corresponding cell module for $A_{\pi}$. Its basis is $\bigcup_{z \in \pi}\left\{\mathbb{L}_{\underline{z}, \mathbf{e}} \mid \mathbf{e} \in \operatorname{Tab}(\underline{z}, y)\right\}$, and there is an $R$-module decomposition

$$
\Delta_{\pi}(y)=\oplus_{z \in \pi} \Delta_{\underline{z}}(y)
$$

and in particular $\operatorname{dim} \Delta_{\pi}(y)=\sum_{z \in \pi} \operatorname{dim} \Delta_{\underline{z}}(y)$. Note that there is no similar formula for $A_{\pi}$. We view (8.14) as a kind of weight space decomposition for $\Delta_{\pi}(y)$ with weight spaces $\Delta_{\underline{z}}(y)$.

We point out that the bilinear form $\langle\cdot, \cdot\rangle_{\pi, y}$ on $\Delta_{\pi}(y)$ is orthogonal with respect to the decomposition in (8.14), as follows directly from the definitions. This is a key observation for the following.

Recall that the ground ring for $A_{\pi}$ is $R$. For a field $\mathbb{k}$ that is made into an $R$-algebra via a homomorphism $R \rightarrow \mathbb{k}$, we obtain as usual a specialized algebra $A_{\pi, \mathbb{k}}:=A_{\pi} \otimes_{R} \mathbb{k}$. This is also a cellular algebra. We now state the result that makes us prefer $A_{\pi}$ over $A_{\underline{w}}$. 
Theorem 8.5. $A_{\pi}$ and $A_{\pi, \mathbb{k}}$ are quasi-hereditary algebras.

Proof: By remark (3.10) of [8] we must show that for $y \in \Lambda_{\pi}$ we have that $\langle\cdot, \cdot\rangle_{\pi, y} \neq 0$. But since $\underline{y}$ is a reduced expression for $y$ we get for $\mathbf{e}=(1,1, \ldots, 1)$ that $\mathbb{L}_{\underline{y}, \mathbf{e}}$ can be chosen as the identity morphism in $\operatorname{End}_{\mathcal{D}}(\underline{y})$ and so $\left\langle\mathbb{L}_{\underline{y}}, \mathbf{e}, \mathbb{L}_{\underline{y}, \mathbf{e}}\right\rangle_{\pi, y}=\left\langle\mathbb{L}_{\underline{y}, \mathbf{e}}, \mathbb{L}_{\underline{y}, \mathbf{e}}\right\rangle_{y}=1$. The Theorem follows from this.

Let $\mathcal{R}:=\mathbb{R}[x]$ or $\mathcal{R}:=\mathbb{Z}_{(p)}$ depending on our choice of realization and define $A_{\pi, \mathcal{R}}, A_{\pi, \mathbb{R}}$ and $A_{\pi, \overline{\mathbb{F}}_{p}}$ correspondingly. By the Theorem, these are all quasi-hereditary algebras with cell modules that we denote $\Delta_{\pi, \mathcal{R}}(y), \Delta_{\pi, \mathbb{R}}(y)$ and $\Delta_{\pi, \overline{\mathbb{F}}_{p}}(y)$. We use the same notation $\langle\cdot, \cdot\rangle_{\pi, y}$ for the bilinear form on each of these modules and introduce the Jantzen type filtrations $\Delta_{\pi, \mathcal{R}}^{i}(y), \Delta_{\pi, \mathbb{R}}^{i}(y)$ and $\Delta_{\pi, \overline{\mathbb{F}}_{p}}^{i}(y)$, mimicking the previous construction.

Let us now turn to the Grothendieck groups. Let $\mathbb{k}$ be either $\mathbb{R}$ or $\overline{\mathbb{F}}_{p}$, depending on the choice of $\mathfrak{h}$. Let $\left\langle A_{\pi, \mathbb{k}}-\bmod \right\rangle$ (resp. $\left.\left\langle A_{\underline{w}, k}-\bmod \right\rangle\right)$ be the Grothendieck groups of finite dimensional $A_{\pi, \mathbb{k}}$-modules (resp. finite dimensional $A_{\underline{w}, \mathbb{k}}$-modules). Because of quasi-heredity, the classes $\left[\Delta_{\pi, \mathbb{k}}(y)\right]$ in $\left\langle A_{\pi, \mathbb{k}}-\bmod \right\rangle$ of the cell modules form a $\mathbb{Z}$-basis for $\left\langle A_{\pi, \mathbb{k}}-\bmod \right\rangle$. On the other hand, the corresponding classes $\left[\Delta_{\underline{w}, \mathbb{k}}(y)\right]$ in $\left\langle A_{\underline{w}, \mathbb{k}}-\bmod \right\rangle$ only form a generating set for $\left\langle A_{\underline{w}, \mathbb{k}}-\bmod \right\rangle$ since $A_{\underline{w}, \mathbb{k}}$ is not quasi-hereditary in general.

Let us consider the following projection map $\varphi_{\underline{w}}$ :

$$
\varphi_{\underline{w}}:\left\langle A_{\pi, \mathbb{k}}-\bmod \right\rangle \rightarrow\left\langle A_{\underline{w}, \mathbb{k}}-\bmod \right\rangle, \quad\left[\Delta_{\pi, \mathbb{k}}(y)\right] \mapsto\left[\Delta_{\underline{w}, \mathbb{k}}(y)\right] .
$$

We need to describe $\varphi_{\underline{w}}$ in a different way. There is a natural diagonal subalgebra $\oplus_{w \in \pi} A_{\underline{w}, \mathbb{k}}$ of $A_{\pi, \mathbb{k}}$ having $A_{\underline{w}, \mathbb{k}}$ as an algebra summand. Let

$$
\tau_{\underline{w}}: A_{\pi, \mathbb{k}}-\bmod \rightarrow A_{\underline{w}, \mathbb{k}}-\bmod
$$

be the composition of the corresponding restriction and idempotent truncation functors. Then $\tau_{\underline{w}}$ induces a $\mathbb{Z}$-module homomorphism between the Grothendieck groups: this is our $\varphi_{\underline{w}}$. Via this description of $\varphi_{\underline{w}}$ and the orthogonality of the decomposition (․14), we get the following compatibility of the Jantzen filtrations

$$
\varphi_{\underline{w}}\left(\left[\Delta_{\pi, \mathbb{k}}^{i}(y)\right]\right)=\left[\Delta_{\underline{w}, \mathbb{k}}^{i}(y)\right] .
$$

Let now $\operatorname{dim}_{\underline{w}}:\left\langle A_{\underline{w}, \mathbb{k}}-\bmod \right\rangle \rightarrow \mathbb{Z},[M] \mapsto \operatorname{dim}_{\mathbb{k}} M$ be the dimension homomorphism and define a $\mathbb{Z}$-module homomorphism $\Phi:\left\langle A_{\pi, \mathbb{k}}-\bmod \right\rangle \rightarrow \oplus_{\underline{w} \in \underline{\pi}} \mathbb{Z}$ by setting the $\underline{w}^{\prime}$ th coordinate equal to the composite $\operatorname{dim}_{\underline{w}} \circ \varphi_{\underline{w}}$.

Lemma 8.6. In the above notation $\Phi:\left\langle A_{\pi, \mathbb{k}}-\right.$ mod $\rangle \rightarrow \oplus_{\underline{w} \in \underline{\pi}} \mathbb{Z}$ is an isomorphism of $\mathbb{Z}$-modules.

Proof: $\quad$ Since $\left\langle A_{\pi, \mathbb{k}}-\bmod \right\rangle$ and $\oplus_{\underline{w} \in \underline{\pi}} \mathbb{Z}$ are free $\mathbb{Z}$-modules of the same rank, it is enough to show that $\Phi$ is surjective. Let $\left(n_{\underline{z}}\right)_{\underline{z} \in \underline{\pi}}$ be an element of $\oplus_{\underline{z} \in \underline{\pi}} \mathbb{Z}$. Choose $\underline{z}_{0}$ satisfying $n_{\underline{z}_{0}} \neq 0$ and $z_{0}$ minimal in $W$ with respect to this. The $\underline{z}_{0}$ 'th component of $\Phi\left(\left[\Delta_{\pi}\left(z_{0}\right)\right]\right)$ is $\operatorname{dim}_{\mathbb{k}} \Delta_{\underline{z}_{0}}\left(z_{0}\right)=1$ and so the $\underline{z}_{0}$ 'th component of $\Phi\left(n_{\underline{z}_{0}}\left[\Delta_{\pi}\left(z_{0}\right)\right]\right)$ is $n_{\underline{z}_{0}}$. Moreover, the $\underline{z}$ 'th component of $\Phi\left(\left[\Delta_{\pi}\left(z_{0}\right)\right]\right)$ is nonzero only if $z_{0} \leq \underline{z}$, or equivalently $z_{0} \leq z$ since $\underline{z} \in \operatorname{rexp}_{s}$. Hence, we can use induction on $\left(n_{\underline{z}}\right)_{\underline{z} \in \underline{\pi}}-\Phi\left(n_{\underline{z}_{0}}\left[\Delta_{\underline{\pi}}\left(z_{0}\right)\right]\right)$ and get that $\left(n_{\underline{z}}\right)_{\underline{z} \in \underline{\pi}} \in i m \Phi$ as claimed.

We are now in position to prove the main Theorems of this section.

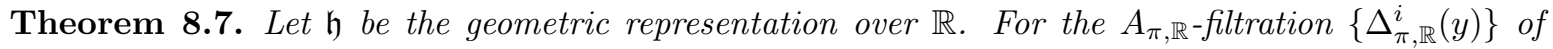
$\Delta_{\pi, \mathbb{R}}(y)$ we have that $\Delta_{\pi, \mathbb{R}}(y) / \Delta_{\underline{\pi}, \mathbb{R}}^{1}(y)$ is nonzero and irreducible and the following sum formula holds in $\left\langle A_{\pi, \mathbb{R}}-\bmod \right\rangle$

$$
\sum_{i>0}\left[\Delta_{\pi, \mathbb{R}}^{i}(y)\right]=\sum_{\beta>0, s_{\beta} y>y}\left[\Delta_{\pi, \mathbb{R}}\left(s_{\beta} y\right)\right] .
$$

Proof: By the construction of the filtration, the first statement follows from the quasi-heredity of $A_{\pi}$.

To show the second statement we get by applying $\Phi$ to the left hand side of (8.18) the element of $\oplus_{\underline{w} \in \underline{\pi}} \mathbb{Z}$ whose $\underline{w}^{\prime}$ th component is $\sum_{i>0} \operatorname{dim} \Delta_{\underline{w}, \mathbb{R}}^{i}(y)$. Applying $\Phi$ to the right hand side of (ㅇ.18), we get the element whose $\underline{w}$ 'th component is $\sum_{\beta>0, s_{\beta} y>y} \operatorname{dim} \Delta_{\underline{w}, \mathbb{R}}\left(s_{\beta} y\right)$ and so by Theorem 8.1 the two sides are equal and the Theorem follows.

Similarly, we have the following Theorem. 
Theorem 8.8. Let $W$ be a Weyl group and let $\mathfrak{h}$ be its Cartan matrix representation over $\overline{\mathbb{F}}_{p}$. For the $A_{\pi, \overline{\mathbb{F}}_{p}}$-filtration $\left\{\Delta_{\pi, \overline{\mathbb{F}}_{p}}^{i}(y)\right\}$ of $\Delta_{\pi, \overline{\mathbb{F}}_{p}}(y)$ we have that $\Delta_{\pi, \overline{\mathbb{F}}_{p}}(y) / \Delta_{\pi, \overline{\mathbb{F}}_{p}}^{1}(y)$ is nonzero and irreducible and the following sum formula holds in $\left\langle A_{\pi, \overline{\mathbb{F}}_{p}}\right.$-mod $\rangle$

$$
\sum_{i>0}\left[\Delta_{\pi, \overline{\mathbb{F}}_{p}}^{i}(y)\right]=\sum_{\beta>0, s_{\beta} y>y} \nu_{p}(\varphi(\beta))\left[\Delta_{\pi, \mathbb{F}_{p}}\left(s_{\beta} y\right)\right]
$$

\section{Applications of the sum formula.}

We indicate in this section, via an example, how to apply formula (8.18) to obtain decomposition numbers for $A_{\pi}$. This is parallel to Jantzen's original calculations for Verma modules and just as in the original setting, the sum formula only gives complete information on the decomposition numbers in certain small cases.

The comparison with Verma modules is strengthened by the following analogue for $A_{\pi}$ of the fact that homomorphisms between Verma modules are injective. For Verma modules this is a consequence of the PBW-Theorem together with the definition of Verma modules as induced modules from a Borel subalgebra. In the $A_{\pi}$-setting, these notions and results are not available and so one must argue in a different way to prove the injectivity. We shall rely on a result of D. Plaza, see [26].

Theorem 9.1. i). Let $W$ be an arbitrary Coxeter group and let $\pi$ an ideal of $W$. Let $\mathfrak{h}$ be the geometric representation of $W$. Suppose that $u, v \in W$ satisfy $u \leq v$. Then there is an embedding of $A_{\pi, \mathbb{R}}$-modules $\Delta_{\pi, \mathbb{R}}(v) \subseteq \Delta_{\pi, \mathbb{R}}(u)$.

ii). Let $W$ be a Weyl group and let $\pi$ be an ideal of $W$. Suppose that $u, v \in W$ satisfy $u \leq v$. Let $\mathfrak{h}$ be the Cartan matrix representation of $W$ with $p>3$. Then there is an embedding of $A_{\pi, \overline{\mathbb{F}}_{p}}$-modules $\Delta_{\pi, \overline{\mathbb{F}}_{p}}(v) \subseteq \Delta_{\pi, \overline{\mathbb{F}}_{p}}(u)$.

Proof: As already mentioned above, in each of the settings i) and ii) we have that $\mathfrak{h}$ is a Soergel realization and so the functor $F: \mathcal{D} \rightarrow \mathbb{B S B i m}$ introduced in section 3 defines an equivalence of categories. For $\underline{w} \in \operatorname{rexp}_{s}$ we define $A_{\underline{w}}^{\mathbb{B S}}:=\operatorname{End}_{\mathbb{B S}}(\underline{w})$ and then $F$ induces an algebra isomorphism $A_{\underline{w}} \rightarrow A_{\underline{w}}^{\mathbb{B S}}$. Let us denote by $\Delta_{\underline{w}}^{\mathbb{B S}}(y)$ the cell module for $A_{\underline{w}}^{\mathbb{B S S}}$ with associated basis $\left\{\mathbb{L}_{\underline{w}, \mathbf{e}}^{\mathbb{B S}} \mid \mathbf{e} \in \operatorname{Tab}(y)\right\}$. Then we have that $F\left(\mathbb{L}_{\underline{w}}, \mathbf{e}\right)=\mathbb{L} \mathbb{L}_{\underline{w}, \mathbf{e}}^{\mathbb{B S}}$. Suppose now that $\underline{u}, \underline{v} \in \operatorname{rexp}_{s}$ satisfy $u \leq v \leq w$ and let $G_{v}^{u} \in$ $\operatorname{Hom}_{\mathbb{B S}}\left(B_{\underline{v}}, B_{\underline{u}}\right)$ be the light leaves morphism associated with Deodhars' distinguished subexpression for $\underline{u}$ in $\underline{v}$. Then in [26] it was proved that the map given by

$$
\Delta_{\underline{w}}^{\mathbb{B S}}(v) \rightarrow \Delta_{\underline{w}}^{\mathbb{B S}}(u), l \mapsto G_{v}^{u} \circ l
$$

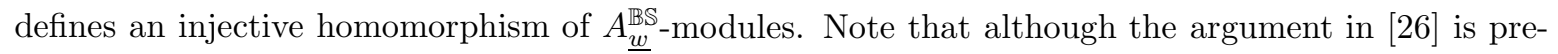
sented for the ground field $\mathbb{R}$, it carries over to characteristic $p$. But then by pulling back via $F$ we get that

$$
\Delta_{\underline{w}}(v) \rightarrow \Delta_{\underline{w}}(u), l \mapsto F^{-1}\left(G_{v}^{u}\right) \circ l
$$

is an injective homomorphism of $A_{\underline{w}}$-modules. The Theorem follows from this by summing over all relevant summands.

Remark. We believe that the Theorem can be proved diagrammatically, without relying on the equivalence $F$.

Let us finish the paper by calculating some decomposition numbers, using Theorem 8.7 and Theorem 8.8. We first choose $\mathbb{k}:=\mathbb{R}$ and $W=\left\langle s_{1}, s_{2}\right\rangle$ of type $A_{2}$, that is

$$
W=\left\{1, s_{1}, s_{2}, s_{1} s_{2}, s_{2} s_{1}, s_{1} s_{2} s_{1}=s_{2} s_{1} s_{2}\right\} .
$$

Let us use $\pi:=W$ and $\underline{\pi}:=\left\{1, s_{1}, s_{2}, s_{1} s_{2}, s_{2} s_{1}, s_{1} s_{2} s_{1}\right\}$.

We associate with this the following alcove geometry 


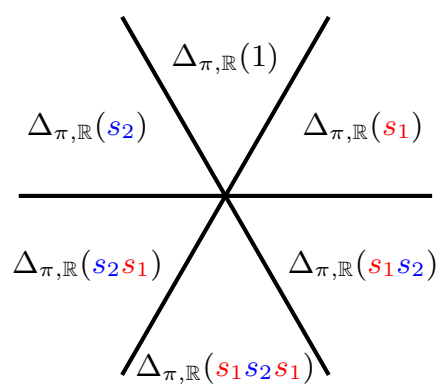

The images of the simple modules $\left[L_{\pi, \mathbb{R}}(y)\right]$, with $y$ running over $\mathrm{W}$, give the canonical basis of $\left\langle A_{\pi, \mathbb{R}}-\bmod \right\rangle$ and by general cellular algebra theory we have that

$$
\left[\Delta_{\pi, \mathbb{R}}(y)\right]=\sum_{y \leq u} d_{y u}^{\mathbb{R}}\left[L_{\pi, \mathbb{R}}(u)\right]
$$

where the $d_{y u}^{\mathbb{R}}$ 's are the decomposition numbers. Let us determine the expansion of all $\left[\Delta_{\pi, \mathbb{R}}(y)\right]$ 's in terms of $\left[L_{\pi, \mathbb{R}}(y)\right]$ 's, or equivalently the decomposition numbers $d_{y u}^{\mathbb{R}}$. As a starting point we get from (9.4) that

$$
\Delta_{\pi, \mathbb{R}}\left(s_{1} s_{2} s_{1}\right)=L_{\pi, \mathbb{R}}\left(s_{1} s_{2} s_{1}\right)
$$

which gives us all the decomposition numbers $d_{y u}^{\mathbb{R}}$ with $y=s_{1} s_{2} s_{1}$. Let us now calculate $\left[\Delta_{\pi, \mathbb{R}}\left(s_{2} s_{1}\right)\right]$. By (9.4) we have that $\left[\Delta_{\pi, \mathbb{R}}\left(s_{2} s_{1}\right)\right]=\left[L_{\pi, \mathbb{R}}\left(s_{2} s_{1}\right)\right]+d\left[L_{\pi, \mathbb{R}}\left(s_{1} s_{2} s_{1}\right)\right]$ for some $d$. The sum formula (8.18) reads in this case

$$
\sum_{i>0}\left[\Delta_{\pi, \mathbb{R}}^{i}\left(s_{2} s_{1}\right)\right]=\left[\Delta_{\pi, \mathbb{R}}\left(s_{1} s_{2} s_{1}\right)\right]=\left[L_{\pi, \mathbb{R}}\left(s_{1} s_{2} s_{1}\right)\right]
$$

Since $\Delta_{\pi, \mathbb{R}}^{1}\left(s_{2} s_{1}\right)=\operatorname{rad}\langle\cdot, \cdot\rangle_{\pi, s_{2} s_{1}}$ this gives us as the only possibility $d=1$, that is

$$
\left[\Delta_{\pi, \mathbb{R}}\left(s_{2} s_{1}\right)\right]=\left[L_{\pi, \mathbb{R}}\left(s_{2} s_{1}\right)\right]+\left[L_{\pi, \mathbb{R}}\left(s_{1} s_{2} s_{1}\right)\right]
$$

Similarly we have

$$
\left[\Delta_{\pi, \mathbb{R}}\left(s_{1} s_{2}\right)\right]=\left[L_{\pi, \mathbb{R}}\left(s_{1} s_{2}\right)\right]+\left[L_{\pi, \mathbb{R}}\left(s_{1} s_{2} s_{1}\right)\right] .
$$

Let us now turn to $\left[\Delta_{\pi, \mathbb{R}}\left(s_{2}\right)\right]$ which by (9.4) can be written as

$$
\left[\Delta_{\pi, \mathbb{R}}\left(s_{2}\right)\right]=\left[L_{\pi, \mathbb{R}}\left(s_{2}\right)\right]+a\left[L_{\pi, \mathbb{R}}\left(s_{1} s_{2}\right)\right]+b\left[L_{\pi, \mathbb{R}}\left(s_{2} s_{1}\right)\right]+c\left[L_{\pi, \mathbb{R}}\left(s_{1} s_{2} s_{1}\right)\right]
$$

for some nonnegative integers $a, b, c$. On the other hand, the sum formula (8.18) gives in this case

$$
\sum_{i>0}\left[\Delta_{\pi, \mathbb{R}}^{i}\left(s_{2}\right)\right]=\left[\Delta_{\pi, \mathbb{R}}\left(s_{1} s_{2}\right)\right]+\left[\Delta_{\pi, \mathbb{R}}\left(s_{2} s_{1}\right)\right]=\left[L_{\pi, \mathbb{R}}\left(s_{1} s_{2}\right)\right]+\left[L_{\pi, \mathbb{R}}\left(s_{2} s_{1}\right)\right]+2\left[L_{\pi, \mathbb{k}}\left(s_{1} s_{2} s_{1}\right)\right]
$$

where we for the second equality used (9.7) and (9.8). This information gives us that $a=b=1$ but it does not determine the value of $c$ since the filtration $\left\{\Delta_{\pi, \mathbb{R}}^{i}\left(s_{2}\right)\right\}$ may have one or two nonzero terms. In fact it has two terms, as one sees via the restriction of the filtration to $\Delta_{s_{1} s_{2} s_{1}, \mathbb{R}}\left(s_{2}\right)$. Indeed we have $\operatorname{dim} \Delta_{s_{1} s_{2} s_{1}, \mathbb{R}}\left(s_{2}\right)=1$ and

$$
\sum_{i>0} \operatorname{dim} \Delta_{s_{1} s_{2} s_{1}, \mathbb{R}}^{i}\left(s_{2}\right)=\operatorname{dim} \Delta_{s_{1} s_{2} s_{1}, \mathbb{R}}\left(s_{1} s_{2}\right)+\operatorname{dim} \Delta_{s_{1} s_{2} s_{1}, \mathbb{R}}\left(s_{2} s_{1}\right)=2
$$

and so there must be two terms in the filtration for $\Delta_{s_{1} s_{2} s_{1}, \mathbb{R}}\left(s_{2}\right)$, and hence also in the filtration for $\Delta_{\pi, \mathbb{R}}\left(s_{2}\right)$. We then deduce that $c=1$.

We now consider $\left[\Delta_{\pi, \mathbb{R}}\left(s_{1}\right)\right]$ which is treated almost the same way. We have that

$$
\left[\Delta_{\pi, \mathbb{R}}\left(s_{1}\right)\right]=\left[L_{\pi, \mathbb{R}}\left(s_{1}\right)\right]+a_{1}\left[L_{\pi, \mathbb{R}}\left(s_{1} s_{2}\right)\right]+b_{1}\left[L_{\pi, \mathbb{R}}\left(s_{2} s_{1}\right)\right]+c_{1}\left[L_{\pi, \mathbb{R}}\left(s_{1} s_{2} s_{1}\right)\right]
$$

and the sum formula gives

$$
\sum_{i>0}\left[\Delta_{\pi, \mathbb{R}}^{i}\left(s_{1}\right)\right]=\left[\Delta_{\pi, \mathbb{R}}\left(s_{2} s_{1}\right)\right]+\left[\Delta_{\pi, \mathbb{R}}\left(s_{1} s_{2}\right)\right]=\left[L_{\pi, \mathbb{R}}\left(s_{2} s_{1}\right)\right]+\left[L_{\pi, \mathbb{R}}\left(s_{1} s_{2}\right)\right]+2\left[L_{\pi, \mathbb{k}}\left(s_{2} s_{1} s_{2}\right)\right]
$$


and so $a_{1}=b_{1}=1$. In order to show that also $c_{1}=1$ we must once again show that there are two nonzero terms in the filtration $\left\{\Delta_{\pi, \mathbb{R}}^{i}\left(s_{1}\right)\right\}$. We here consider $\Delta_{s_{1} s_{2} s_{1}, \mathbb{R}}\left(s_{1}\right)$ which is of dimension two, via the following light leaves basis

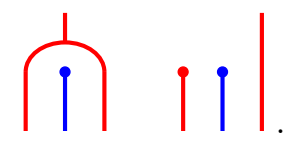

With respect to this basis, the matrix of the bilinear form $\langle\cdot, \cdot\rangle_{s_{1}}$ is

$$
\left[\begin{array}{cc}
-1 & \alpha_{2} \\
\alpha_{2} & \alpha_{1} \alpha_{2}
\end{array}\right] \sim\left[\begin{array}{cc}
-1 & 0 \\
0 & \left(\alpha_{1}+\alpha_{2}\right) \alpha_{2}
\end{array}\right] \stackrel{\varphi}{\longrightarrow}\left[\begin{array}{cc}
-1 & 0 \\
0 & 2 x^{2}
\end{array}\right]
$$

where $\sim$ refers to the diagonalization procedure indicated in Theorem 8.1 . The $2 x^{2}$ entry of this matrix tells us that there are two nonzero terms in the filtration for $\Delta_{s_{1} s_{2} s_{1}, \mathbb{R}}\left(s_{1}\right)$, and so there are also two nonzero terms in the filtration for $\Delta_{\pi, \mathbb{R}}\left(s_{1}\right)$. We get from this that $c_{1}=1$ as claimed.

Finally, using similar arguments we have that

$$
\left[\Delta_{\pi, \mathbb{R}}(1)\right]=\left[L_{\pi, \mathbb{R}}(1)\right]+\left[L_{\pi, \mathbb{R}}\left(s_{1}\right)\right]+\left[L_{\pi, \mathbb{R}}\left(s_{2}\right)\right]+\left[L_{\pi, \mathbb{R}}\left(s_{1} s_{2}\right)\right]+\left[L_{\pi, \mathbb{R}}\left(s_{2} s_{1}\right)\right]+\left[L_{\pi, \mathbb{R}}\left(s_{1} s_{2} s_{1}\right)\right] .
$$

Using Theorem 8.7 one can also carry out these calculations in the characteristic $p>3$ cases. In fact, one gets in these cases the same decomposition numbers.

In the language of $p$-canonical bases, see [15, we get that for $A_{2}$ the $p$-canonical basis coincides with the Kazhdan-Lusztig basis. This is already proved in [15] using very different methods.

In view of the results and conjectures of [29, we note that it would be very interesting to generalize our results to the anti-spherical module of affine Weyl groups.

\section{References}

[1] H.H. Andersen, A sum formula for tilting filltrations, Journal of Pure and Applied Algebra 152 (2000), 17-40.

[2] H.H. Andersen, Filtrations of cohomology modules for Chevalley groups, Annales scientifiques de l'É.N.S. $4^{e}$ série, tome 16, no 4 (1983), 495-528.

[3] B. Elias, The two-color Soergel calculus, Compositio Mathematica 152 (2015), 327-398.

[4] B. Elias, G. Williamson, The Hodge theory of Soergel bimodules, Annals of Mathematics (2) 180 (2014), no. 3, 1089-1136.

[5] B. Elias, G. Williamson, Soergel calculus, Representation Theory 20 (2016), 295-374.

[6] B. Elias, G. Williamson, Diagrammatics for Coxeter groups and their braid groups, Quantum Toplogy, 8(3) (2017), 413-457.

[7] B. Elias, M. Khovanov, Diagrammatics for Soergel Categories, International Journal of Mathematics and Mathematical Sciences 2010 (2010), 58 pages.

[8] J. J. Graham, G. I. Lehrer, Cellular algebras, Inventiones Mathematicae 123 (1996), 1-34.

[9] J. Humphreys, Representations of semisimple Lie algebras in the BGG category O, (2008), Graduate Studies in Mathematics 94, Providence, R.I.: American Mathematical Society, ISBN 978-08218-4678-0.

[10] J. E. Humphreys, Reflection groups and Coxeter groups, volume $\mathbf{2 9}$ of Cambridge Studies in Advanced Mathematics. Cambridge University Press, Cambridge, 1990.

[11] G. D. James, G.E. Murphy, The Determinant of the Gram Matrix for a Specht Module, Journal of Algebra 59 (1979). 
[12] J. C. Jantzen, Moduln mit einem höchsten Gewicht, Lecture Notes in Mathematics 750, Berlin, New York: Springer-Verlag, doi:10.1007/BFb0069521, ISBN 978-3-540-09558-3.

[13] A. A. Jucys, On the Young operators of symmetric groups, Litousk. Fiz Sb. 6 (1966), 163-180.

[14] A. A. Jucys, Factorisation of Young's projection operators of symmetric groups, Litousk. Fiz. Sb. 11 (1971), l-10.

[15] L. T. Jensen, G. Williamson, The p-Canonical Basis for Hecke Algebras, in Categorification in Geometry, Topology and Physics, 333-361, Contemp. Math. 583 (2017).

[16] N. Libedinsky, Sur la catégorie des bimodules de Soergel, Journal of Algebra 320(7) (2008), 26752694.

[17] N. Libedinsky, Gentle introduction to Soergel bimodules I: The basics, Sao Paulo Journal of Mathematical Sciences, 13(2) (2019), 499-538.

[18] N. Libedinsky, Équivalences entre conjectures de Soergel, Journal of Algebra 320 (2008), 26952705.

[19] N. Libedinsky, Light leaves and Lusztig's conjecture, Advances in Mathematics 280 (2015), 772807.

[20] A. Mathas, Hecke algebras and Schur algebras of the symmetric group, Univ. Lecture Notes, 15, A.M.S., Providence, R.I., 1999.

[21] A. Mathas, Seminormal forms and Gram determinants for cellular algebras, J. Reine Angew. Math., 619 (2008), 141-173.

[22] G. E. Murphy, A New Construction of Young's Seminormal Representation of the Symmetric Groups, Journal of Algebra 69 (1981), 287-297.

[23] G. E. Murphy, The Idempotents of the Symmetric Groups and Nakayama's Conjecture, Journal of Algebra 81 (1983), 258-265.

[24] G. E. Murphy, On the Representation Theory of the Symmetric Groups and Associated Hecke Algebras, Journal of Algebra 152 (1992), 492-513.

[25] G. E. Murphy, The Representations of Hecke Algebras of type $A_{n}$, Journal of Algebra 173 (1995), $97-121$.

[26] D. Plaza, Graded cellularity and the Monotonicity Conjecture, Journal of Algebra, 473 (2017), 324-351.

[27] D. Plaza, Categorification of a recursive formula for Kazhdan-Lusztig polynomials, Comm. in Algebra, 44 (2016), no. 10, 4354-4377.

[28] S. Ryom-Hansen, Projective modules for the symmetric group and Young's seminormal form, Journal of Algebra 439 (2015), 515-541.

[29] S. Riche, G. Williamson, Tilting modules and the p-canonical basis, Astérisque 397, (2018), ix +184 .

[30] N. N. Shapovalov, On bilinear form on universal enveloping algebra of a complex semisimple Lie algebra, Funct. Anal. Appl. 6 (1972), 307-312.

[31] W. Soergel, Kazhdan-Lusztig-Polynome und unzerlegbare Bimoduln über Polynomringen, J. Inst. Math. Jussieu 6 (2007), no. 3, 501-525.

[32] W. Soergel. Kategorie $\mathcal{O}$, perverse Garben und Moduln über den Koinvarianten zur Weylgruppe. J. Amer. Math. Soc., 3(2) (1990), 421-445,

[33] B. W. Westbury, Invariant tensors and cellular categories, Journal of Algebra, 321(11) 2009, 3563-3567. 
[34] G. Williamson, Schubert calculus and torsion explosion (with an appendix by A. Kontorovich, P. McNamara and G. Williamson), J. Amer. Math. Soc., 30(4), 2017, 1023-1046.

Instituto de Matemática y Física, Universidad de Talca, Chile, steen@inst-mat.utalca.Cl. 